PREPARED FOR SUBMISSION TO JCAP

\title{
Non-linear evolution of the cosmic neutrino background
}

\section{Francisco Villaescusa-Navarro, ${ }^{a}$ Simeon Bird, ${ }^{b}$ Carlos Peña-Garay, ${ }^{c}$ Matteo Viel ${ }^{a, d}$}

\author{
${ }^{a}$ INAF/Osservatorio Astronomico di Trieste, Via Tiepolo 11, 34143, Trieste, Italy \\ ${ }^{b}$ Institute for Advanced Study, 1 Einstein Drive, Princeton, NJ, 08540, USA \\ ${ }^{c}$ Instituto de Física Corpuscular, CSIC-UVEG, E-46071, Paterna, Valencia, Spain \\ ${ }^{d}$ INFN sez. Trieste, Via Valerio 2, 34127 Trieste, Italy \\ E-mail: villaescusa@oats.inaf.it,spb@ias.edu,penya@ific.uv.es, viel@oats.inaf.it
}

\begin{abstract}
We investigate the non-linear evolution of the relic cosmic neutrino background by running large box-size, high resolution N-body simulations which incorporate cold dark matter (CDM) and neutrinos as independent particle species. Our set of simulations explore the properties of neutrinos in a reference $\Lambda$ CDM model with total neutrino masses between $0.05-0.60 \mathrm{eV}$ in cold dark matter haloes of mass $10^{11}-10^{15} h^{-1} \mathrm{M}_{\odot}$, over a redshift range $z=0-2$. We compute the halo mass function and show that it is reasonably well fitted by the Sheth-Tormen formula, once the neutrino contribution to the total matter is removed. More importantly, we focus on the CDM and neutrino properties of the density and peculiar velocity fields in the cosmological volume, inside and in the outskirts of virialized haloes. The dynamical state of the neutrino particles depends strongly on their momentum: whereas neutrinos in the low velocity tail behave similarly to CDM particles, neutrinos in the high velocity tail are not affected by the clustering of the underlying CDM component. We find that the neutrino (linear) unperturbed momentum distribution is modified and mass and redshift dependent deviations from the expected Fermi-Dirac distribution are in place both in the cosmological volume and inside haloes. The neutrino density profiles around virialized haloes have been carefully investigated and a simple fitting formula is provided. The neutrino profile, unlike the cold dark matter one, is found to be cored with core size and central density that depend on the neutrino mass, redshift and mass of the halo, for halos of masses larger than $\sim 10^{13.5} h^{-1} \mathrm{M}_{\odot}$. For lower masses the neutrino profile is best fitted by a simple power-law relation in the range probed by the simulations. The results we obtain are numerically converged in terms of neutrino profiles at the $10 \%$ level for scales above $\sim 200 h^{-1} \mathrm{kpc}$ at $z=0$, and are stable with respect to box-size and starting redshift of the simulation. Our findings are particularly important in view of upcoming large-scale structure surveys, like Euclid, that are expected to probe the non-linear regime at the percent level with lensing and clustering observations.
\end{abstract}




\section{Contents}

1 Introduction 1

2 The simulations $\quad 2$

3 Results

3.1 The non-linear density field 4

$\begin{array}{ll}3.2 & \text { The non-linear velocity field }\end{array}$

$\begin{array}{ll}3.3 \text { The halo mass function } & 8\end{array}$

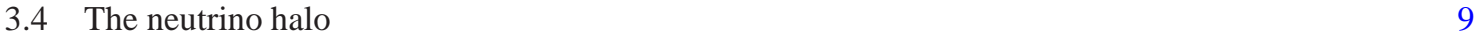

$\begin{array}{llr}3.4 .1 \quad \text { Fitting function } & 13\end{array}$

$\begin{array}{ll}3.4 .2 \text { Convergence tests } & 15\end{array}$

$\begin{array}{ll}\text { 3.5 Neutrino velocity distribution within CDM haloes } & 17\end{array}$

4 Conclusions $\quad 17$

A Impact of the CDM halo environment $\quad 19$

B Fitting formula parameters: dependence with $M_{\mathrm{CDM}}$ and $\Sigma_{i} m_{\nu_{i}}$

\section{Introduction}

The standard Big Bang cosmology predicts the existence of a relic particle radiation in the form of neutrinos, the cosmic neutrino background. In the primordial plasma, the three standard model neutrino flavors are in thermal equilibrium with photons, electrons and positrons and their momentum distribution followed the Fermi-Dirac distribution. When the temperature of the universe drops to $\sim 10^{10} \mathrm{~K}(\sim 1 \mathrm{MeV})$, the expansion rate becomes larger than the rate of neutrino interactions, triggering the decoupling of the neutrinos from the rest of the plasma [1-3]. At the time of neutrino decoupling, neutrino momenta are much larger than their masses, and thus, the number density of neutrinos with momentum between $p$ and $p+d p$ is:

$$
n(p) d p=\frac{4 \pi g_{\nu}}{(2 \pi \hbar c)^{3}} \frac{p^{2} d p}{e^{\frac{p}{k_{B} T_{\nu}}}+1},
$$

where $g_{\nu}$ is the number of neutrino spin states, $k_{B}$ is the Boltzmann constant and $T_{\nu}=T_{\nu}\left(z_{\text {dec }}\right)$ is the temperature of the universe at the neutrino decoupling time ${ }^{1}$. Once neutrinos decouple, their momentum is redshifted as $1 /(1+z)$ and their distribution is given by Eq. 1.1 with $T_{\nu}=T_{\nu}(z)=T_{\nu}\left(z_{\text {dec }}\right)(1+z) /\left(1+z_{\text {dec }}\right)$. It can be shown (see for example [4]) that current neutrino and CMB temperatures are related through $T_{\nu}(z=$ $0)=\left(\frac{4}{11}\right)^{1 / 3} T_{\gamma}(z=0)$ (a small correction to this formula arises when taking into account that some neutrinos are still weakly coupled at the electron-positron annihilation [5]). Equation 1.1 represents the unperturbed momentum distribution of the cosmic neutrino background, i.e. in deriving it we have considered only the redshift of neutrino momentum due to the expansion of the universe. The number density and mean thermal velocity of cosmic neutrinos (neutrinos plus antineutrinos) can be computed from equation 1.1, resulting in $\bar{n}_{\nu}(z) \cong 113(1+z)^{3} \frac{\nu}{\mathrm{cm}^{3}}$ and $\bar{V}_{\nu}(z) \cong 160(1+z)\left(\frac{\mathrm{eV}}{m_{\nu}}\right) \mathrm{km} / \mathrm{s}$, respectively.

We have indirect evidence of the existence of the relic neutrino background. The three light neutrino species contribute to the radiation energy density, changing the expansion rate of the Universe and the time of matter-radiation equality. This in turn affects both Big Bang nucleosynthesis, and thus the primordial abundances of light elements, and the cosmic microwave background (CMB) anisotropies. Finally, late-time freestreaming of the neutrinos suppresses the growth of matter perturbations [1-3]. This effect is well understood

\footnotetext{
${ }^{1}$ Note that we have made the approximation $E_{\nu}\left(z_{d e c}\right)=\sqrt{m_{\nu}^{2}+p_{\nu}^{2}\left(z_{d e c}\right)} \cong p_{\nu}\left(z_{d e c}\right)$.
} 
at the linear level, but on non-linear scales other theoretical or numerical tools have to be used, such as N-body simulations [6-15], perturbation theory [16, 17] or semi-analytical methods [18-23].

From the particle physics side, the discovery of flavor conversion in neutrino experiments, coined neutrino oscillations, implies that at least two of the three neutrino species are massive, with minimal masses of about 9 and $50 \mathrm{meV}$ [24]. From the cosmological side, an important and competitive way to constrain the mass and number of cosmological neutrinos is offered by Large-Scale Structure (LSS) data. The clustering properties of cold dark matter (CDM) and neutrinos (hot dark matter) are very different. Neutrino clustering is strongly influenced by the clustering of the dominant CDM component, but the much larger thermal velocities of neutrinos, as compared to the CDM one, leads to a suppression of the total clustering on small scales and could produce neutrino overdensities in regions where the CDM density is high, in a mass and redshift dependent fashion.

Present cosmological data put an upper limit of $\sim 0.30 \mathrm{eV}$, at the $2 \sigma$ confidence level, on the total neutrino mass by using large LSS data such as SDSS $^{2}$ luminous red galaxies, CFHTLS ${ }^{3}$ or WiggleZ ${ }^{4}$ (e.g. [25-31]). With the notable exceptions of the work of [29], based on CFHTLS and VIPERS ${ }^{5}$ galaxy clustering data, and the analysis of [32], that rely on the Lyman- $\alpha$ forest $\left(\sum_{i} m_{\nu_{i}}<0.17 \mathrm{eV}\right)$, these constraints use only the information contained in the linear regime.

Therefore, the sum of neutrino masses is constrained within an order of magnitude and the model with massless neutrinos has to be modified by a specific model of massive neutrinos with two hierarchical mass splittings. Incoming data analysis of the CMB $[33,34]$ will further improve the significance on the existence of relic neutrinos and with the LSS data in the linear regime may sign to a positive signal on the total mass of neutrinos (e.g. [35-38]). These cosmological observations are thereby very important. Though we do not have a definitive theory of flavor, the total mass determination would allow to distinguish between two large groups of models: degenerate vs hierarchical mass models. More importantly, a large total neutrino mass (in the sensitivity range of running CMB and LSS observations), will fix the predictions of majorana neutrinos in the minimal extension of the standard model of particles physics, within the range of sensitivity of next generation neutrinoless double beta decay experiments [39], and therefore, would allow the experiment to distinguish whether neutrinos are their own antiparticles, and unambiguously imply the existence of a new high energy scale in physics [40].

While some recent works have explored the potential of observables sensitive to the neutrino mass in the nonlinear regime $[19,20]$ by other methods, high resolution large box-size N-body simulations are the most accurate way to describe non-linear gravitational clustering. Previous works using N-body simulations including neutrinos have examined the effect on the matter power spectrum $[8,11,13,14]$. In this work, we will address more closely the density and peculiar velocity fields, similarly to [41] but with improved simulations and a more extensive analysis that brackets a large dynamical and redshift range. We will study the evolution of the non-linear distribution of neutrinos in the whole simulated box, as well as the neutrino properties inside and around virialized haloes. In particular, we will compute and characterize the neutrino density profile around massive CDM halos, providing the reader a fitting formula that reproduces them. Also, we will compute the neutrino momentum distribution and determine how closely it adheres to equation 1.1.

The paper is organized as follows. In Section 2 we describe our N-body simulations. The evolution of the neutrinos and dark matter density and peculiar velocity fields, the halo mass functions and the properties inside and in the neighborhood of dark matter haloes will be addressed in Section 3. This section will contain the main results of the paper and present fitting formula for several different quantities (in the appendix we will show the dependence of some of the physical quantities on the environment). Finally, in Section 4, we summarize the main results of this work and present future perspectives.

\section{The simulations}

We use particle-based N-body simulations containing CDM and neutrino particles, performed using a modified version of the TreePM code GADGET-3, as described in detail in [11, 13, 42]. Neutrinos are treated as dark

\footnotetext{
${ }^{2}$ http://www.sdss.org/

${ }^{3}$ http://www.cfht.hawaii.edu/Science/CFHLS/

${ }^{4}$ http://wigglez.swin.edu.au/site/

${ }^{5}$ http://vipers.inaf.it/
} 


\begin{tabular}{|c|c|c|c|c|c|c|}
\hline Name & $\Sigma_{i} m_{\nu_{i}}(\mathrm{eV})$ & Box $\left(h^{-1} \mathrm{Mpc}\right)$ & $N_{\mathrm{DM}}^{1 / 3}$ & $N_{\nu}^{1 / 3}$ & $z_{i}$ & $\sigma_{8}(z=0)$ \\
\hline \hline H60 & 0.60 & 1000 & 512 & 1024 & 19 & 0.6760 \\
\hline L60 & 0.60 & 500 & 512 & 1024 & 99 & 0.6760 \\
\hline L45 & 0.45 & 500 & 512 & 1024 & 99 & 0.7133 \\
\hline L30 & 0.30 & 500 & 512 & 1024 & 99 & 0.7531 \\
\hline L15 & 0.15 & 500 & 512 & 1024 & 99 & 0.7947 \\
\hline L0 & 0.00 & 500 & 512 & 0 & 99 & 0.8325 \\
\hline S60 & 0.60 & 100 & 512 & 1024 & 99 & 0.6760 \\
\hline S30 & 0.30 & 100 & 512 & 1024 & 99 & 0.7531 \\
\hline LL60 & 0.60 & 500 & 512 & 512 & 99 & 0.6760 \\
\hline LL45 & 0.45 & 500 & 512 & 512 & 99 & 0.7133 \\
\hline LL5 & 0.05 & 500 & 512 & 512 & 99 & 0.8120 \\
\hline
\end{tabular}

Table 1. Summary of simulation parameters. $m_{\nu_{i}}$ is the mass of a single neutrino species, and $\Sigma_{i} m_{\nu_{i}}$ the total neutrino mass. Cosmological parameters are the same for all simulations and are given in the text. $\Omega_{\mathrm{M}}$ is kept constant, so that for an increase in neutrino mass $\Omega_{\mathrm{cdm}}$ decreased and the neutrinos make up an increased fraction of the total dark matter.

matter particles, with a large initial thermal velocity drawn from the Fermi-Dirac distribution of Eq. 1.1. The time-step used by the code is set by the CDM only, and is not affected by the neutrino particles. The force on the neutrinos includes the contribution from the short-range tree: this is in contrast to [11], where the neutrino force was computed using the long-range particle-mesh only. We found that the short-range tree force is required to properly resolve the clustering of the neutrinos in the center of massive halos at low redshift. However, for simulation S30 only (see text below), for reasons of performance the tree is disabled for the neutrinos between $z=99$ and $z=20$.

Our initial conditions are produced from transfer functions generated by CAMB [53], using our own version of N-GenICs modified to use second order Lagrangian perturbation theory [43] for the CDM particles ${ }^{6}$. The transfer function used for our cold dark matter (CDM) particles is a weighted average of the linear theory transfer functions for CDM and baryons, to account for the slight difference between them [44]. For the neutrinos initial conditions we use the Zel'dovich approximation [45], with identical initial random phase information to the CDM one, in order to simulate adiabatic initial conditions. Our cosmological parameters are the following: $\Omega_{\mathrm{b}}=0.05, \Omega_{\mathrm{M}}=\Omega_{\mathrm{CDM}}+\Omega_{\mathrm{b}}+\Omega_{\nu}=0.2708, \Omega_{\Lambda}=0.7292, n_{\mathrm{s}}=1.0, h=0.703$, $A_{\mathrm{S}}=2.43 \times 10^{-9}$, which are roughly in agreement with [46]. The massless neutrino case has a $\sigma_{8}=0.8325$ which is also in reasonable agreement with LSS data. The starting redshift of most of our simulations is set $z=99$. However, we check that our results are insensitive to this value with simulations started at later times $(z=49$ and $z=19$ ). We used a variety of box sizes and particle numbers to ensure our results were independent of both unresolved large-scale modes and unresolved small-scale structure. In particular we simulate three different boxes of linear size 1000, 500 and $100 \mathrm{com} . h^{-1} \mathrm{Mpc}$ with always the same number of CDM particles $\left(512^{3}\right)$. For the neutrinos we use two different numbers of total neutrino particles in order to address the neutrino shot-noise $\left(512^{3}\right.$ and $\left.1024^{3}\right)$ and simulate five different realizations, each of them corresponding to three degenerate neutrino species with $\Sigma_{i} m_{\nu_{i}}=0.15,0.30,0.45,0.60 \mathrm{eV}$. We also consider the case with $\Sigma_{i} m_{\nu_{i}}=0.05 \mathrm{eV}$, for which we produce an initial power spectrum considering only one massive neutrino species.

The gravitational softening length is set to $1 / 40$ of the mean inter-particle spacing for the neutrinos, and the number of cells per side in the particle-mesh grid is $N_{\nu}^{1 / 3}$. The parameters of our simulations are shown in Table 1. We also run a simulation identical to L60, but with a different random seed for the initial structure field, to verify that our results were insensitive to the realisation of cosmic structure. The total CPU time consumption for the neutrino simulations are between 15000-45000 hrs.

\footnotetext{
${ }^{6}$ Our initial conditions generator is freely available at http://github.com/sbird/S-GenIC.
} 

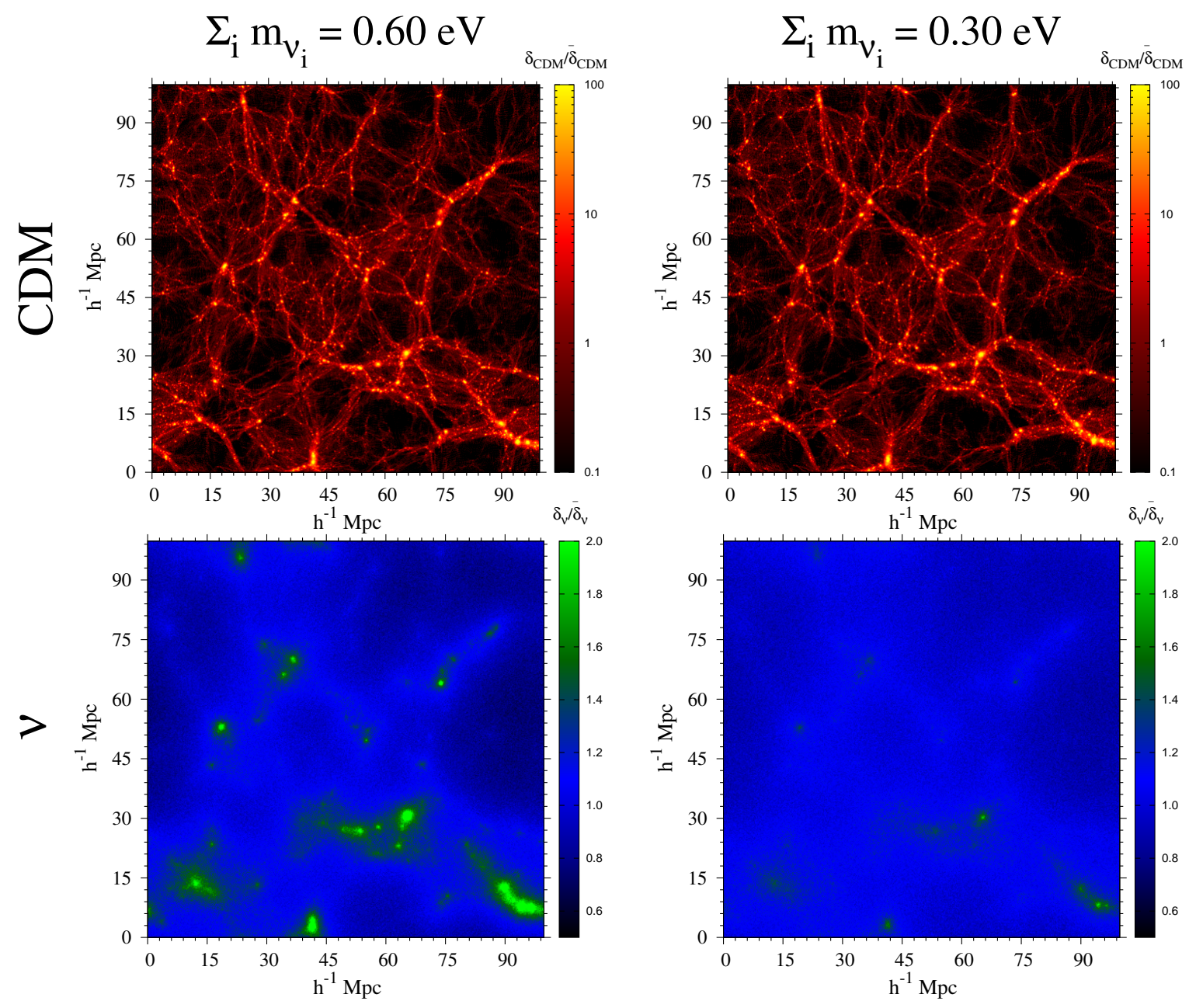

Figure 1. Slice of thickness $5 \mathrm{com} . h^{-1} \mathrm{Mpc}$ through the density field of CDM and neutrinos. The upper panels show a slice of the CDM density field extracted from a N-body simulation with neutrinos of masses $\Sigma_{i} m_{\nu_{i}}=0.60 \mathrm{eV}$ (left column) and $\Sigma_{i} m_{\nu_{i}}=0.30 \mathrm{eV}$ (right column). The bottom panels show the neutrino density field in the same slices of the upper panels.

\section{Results}

In this Section we analyse the suite of simulations and present our main results ${ }^{7}$. Firsly, we will focus on nonlinear properties on large scales, studying the probability distribution function of both the non-linear density field and the non-linear peculiar velocity field. Then we will consider dark matter haloes and compute the mass function for different cosmologies with different neutrino masses at several redshifts. We will also study in detail the clustering of cosmological relic neutrinos within the gravitational potential wells of CDM haloes, providing the reader with a fitting function for the neutrino density profiles.

\subsection{The non-linear density field}

In Fig. 1 we show a slice of thickness 5 com. $h^{-1} \mathrm{Mpc}$ for the density fields of the CDM component and neutrinos of masses $\Sigma_{i} m_{\nu_{i}}=0.60 \mathrm{eV}$ (left panels) and $\Sigma_{i} m_{\nu_{i}}=0.30 \mathrm{eV}$ (right panels). As expected, the regions around which neutrinos tend to cluster are those where the density of CDM is large. This can be seen

\footnotetext{
${ }^{7}$ Several movies showing the distributions of $\mathrm{CDM}$ and $\nu$ are available at http://som.ific.uv.es/movies
} 

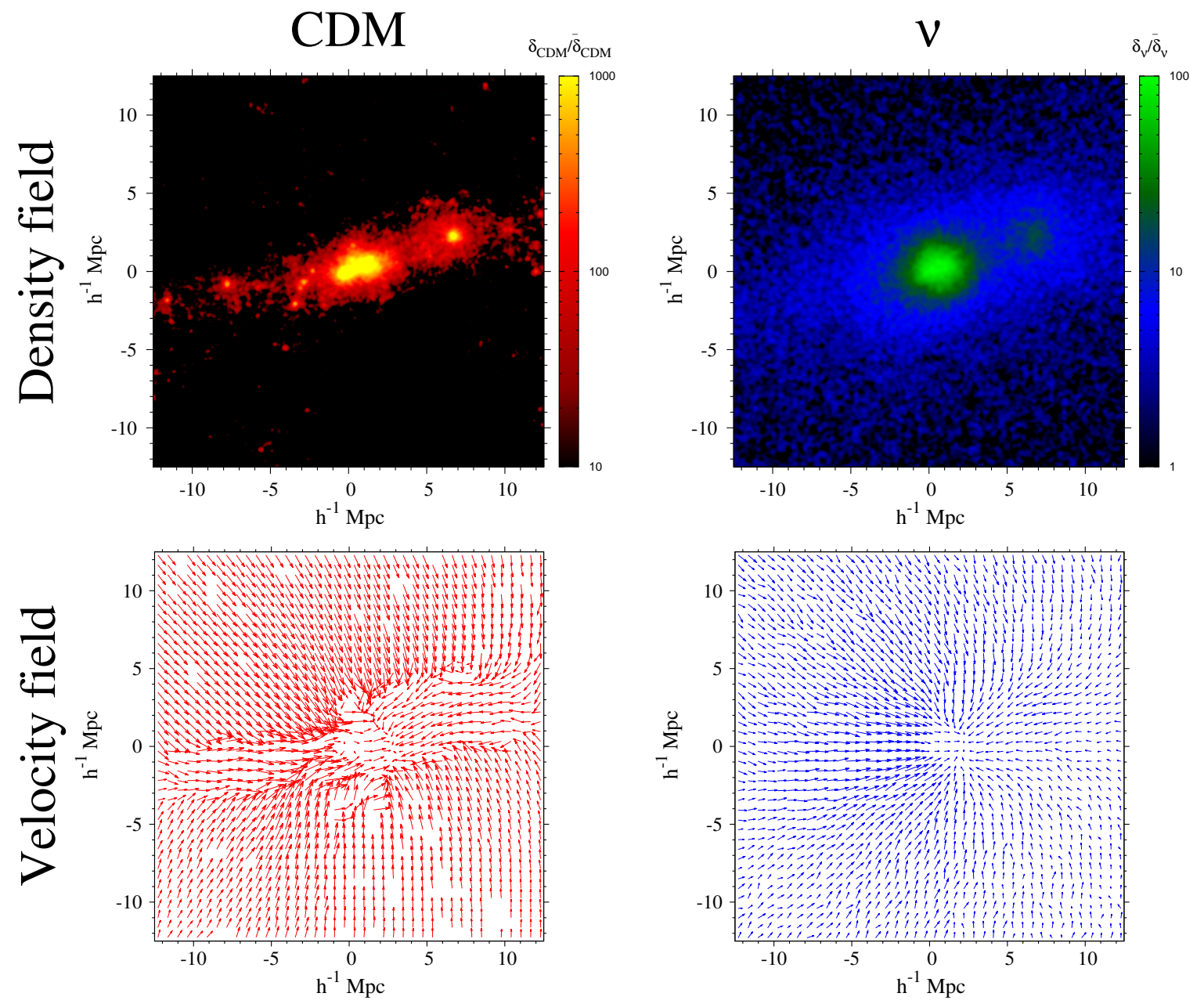

Figure 2. Distribution of densities and peculiar velocities around the most massive CDM halo as extracted from the simulation L60. The position of the center of the halo is $(0,0) h^{-1} \mathrm{Mpc}$ in all panels. Upper left: CDM density distribution. Upper right: neutrino density distribution. Bottom left: CDM peculiar velocities. Bottom right: neutrino peculiar velocities. In all the panels the quantities have been projected over the same plane. All figures have been computed using the CIC interpolation scheme in a slice of linear size $25 h^{-1} \mathrm{Mpc}$ having a thickness of $4 h^{-1} \mathrm{Mpc}$ (comoving units).

even more clearly in Fig. 2, where we show the density and peculiar velocity fields, for the CDM and the cosmological neutrinos, in the neighborhood of the most massive halo present in simulation L60. As we shall see, the neutrino clustering depends on the total neutrino mass, the mass of the haloes and redshift.

We now turn to a more detailed analysis of the density field by computing the value of the density field in a grid of $500 \times 500 \times 500$ points using the cloud-in-cell $(\mathrm{CIC})$ interpolation. This is done for the density fields of both the CDM and the neutrinos. The simulations we have used are LL45 and LL60 (which have the same number of CDM and neutrino particles). In Figure 3 we plot, as a function of the overdensity, $\delta=\rho / \bar{\rho}$, the fraction of grid points whose overdensities are between $\delta$ and $\delta+\triangle \delta$, per $\triangle \delta$, at three different redshifts: $z=0,1,3$. The results for the cosmological models with $\Sigma_{i} m_{\nu_{i}}=0.45 \mathrm{eV}$ and $\Sigma_{i} m_{\nu_{i}}=0.60 \mathrm{eV}$ are plotted on the left and right panels, respectively.

As envisaged, the distribution of the CDM density field stretches out at lower redshift as the cosmic structure form: voids become emptier at the expenses of overdense regions that become denser and denser with decreasing redshift. We note that the distributions for the CDM component are not exactly equal in the two cosmological models. In the cosmological model with neutrino masses equal to $\Sigma_{i} m_{\nu_{i}}=0.45 \mathrm{eV}$ (left 

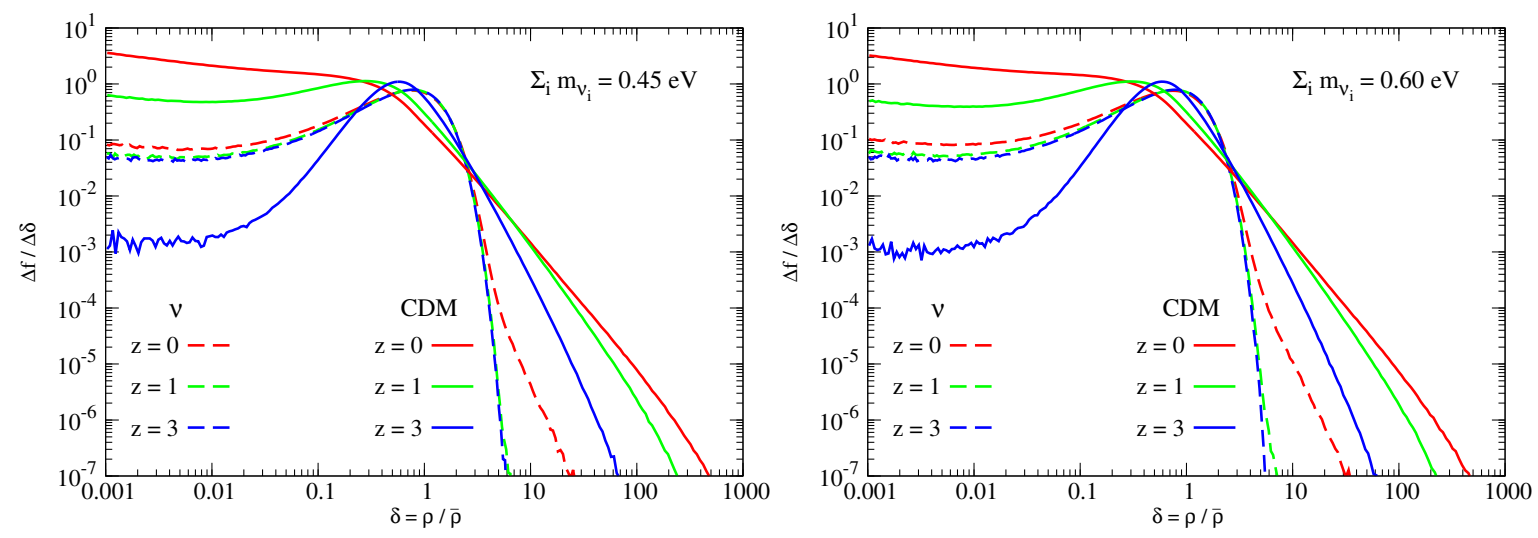

Figure 3. Redshift evolution of the non-linear density field computed over the whole cosmological simulated volume. We use the CIC interpolation scheme to compute the value of density field, for CDM and neutrinos, in a regular grid of $500 \times 500 \times 500$ points. We show the fraction of grid points with overdensities between $\delta$ and $\delta+\triangle \delta$, per $\triangle \delta$, at three different redshifts $z=0,1,3$ (red, green and blue curves, respectively) for CDM (solid lines) and neutrinos (dashed lines). The left panel shows the results for the simulation LL45, while the right panel corresponds to the simulation LL60.

panel), the number of grid points with high or low overdensities is slightly larger than in the cosmology with $\Sigma_{i} m_{\nu_{i}}=0.60 \mathrm{eV}$. This is due to their different power spectra, as reflected in the value of $\sigma_{8}=0.7133(0.45$ $\mathrm{eV}$ ) and $\sigma_{8}=0.676(0.60 \mathrm{eV})$. In contrast, the distribution of neutrinos displays a much slower evolution than CDM: while the fraction of grid points with low CDM densities grows rapidly, the fraction of grid points with low neutrino densities barely changes with time. On the other hand, the fraction of grid points with large neutrino overdensities increases significantly between $z=1$ and $z=0$, reflecting the fact that nonlinear neutrino clustering takes place only at low redshift, as also found by [15]. We also find that the neutrino density field evolves more slowly for lower neutrino masses: this happens because lighter neutrinos have higher thermal velocities which prevents them from clustering into CDM haloes or being excluded from the interior of cosmological voids. It is important to remark that the results we show in Fig. 3 are not fully numerically converged. By repeating the same procedure for the simulations L45 and L60 we find that only the high density tail, $\delta \gtrsim 5$, is converged. The aim of a calculation like the one presented in this Section is to qualitatively show the reader the different redshift evolution of the CDM and cosmic neutrino density fields.

\subsection{The non-linear velocity field}

We now focus on the time evolution of the non-linear peculiar velocity field of both CDM and neutrinos. As in the case of the non-linear density field, we expect the velocity fields of CDM and neutrinos to behave differently. On one hand, by definition, the CDM has negligible peculiar velocities initially. We would thus expect that on average, the CDM particles will increase their peculiar velocities since, among others, they will cluster within virialized haloes and escape from cosmological voids. On the other hand, neutrinos should behave in the opposite way: at high redshift neutrinos have very large thermal velocities, and if we neglect effects associated with gravity, such as neutrino clustering, their thermal velocities should drop with time as $1 /(1+z)$.

We now test the validity of the above simple arguments using N-body simulations. For a given simulation snapshot, we compute the modulus of the peculiar velocity of all, CDM and neutrino, particles in the box. We then calculate the fraction of particles, of each type, whose peculiar velocity modulus lies between $V_{\text {pec }}$ and $V_{\text {pec }}+\triangle V_{\text {pec }}$, per $\triangle V_{\text {pec }}$. We show the results in the Fig. 4 for the simulation L60 (left panel) and L45 (right panel). We do not find significant differences when we use the lower neutrino resolution simulations LL60 and LL45, except in the very low velocity tail $\left(V_{\text {peculiar }} \lesssim 10 \mathrm{~km} / \mathrm{s}\right)$ and thus the results we show in Fig. 4 are numerically converged. Solid lines show the results for the CDM particles at $z=3$ (blue), $z=1$ (green) and $z=0$ (red). On average, CDM particles increase the modulus of their peculiar velocities as time passes and structure formation progresses. The results for the neutrino particles are represented by the thick longdashed curves and their behavior is opposite to the CDM one: on average, neutrinos decrease their peculiar 

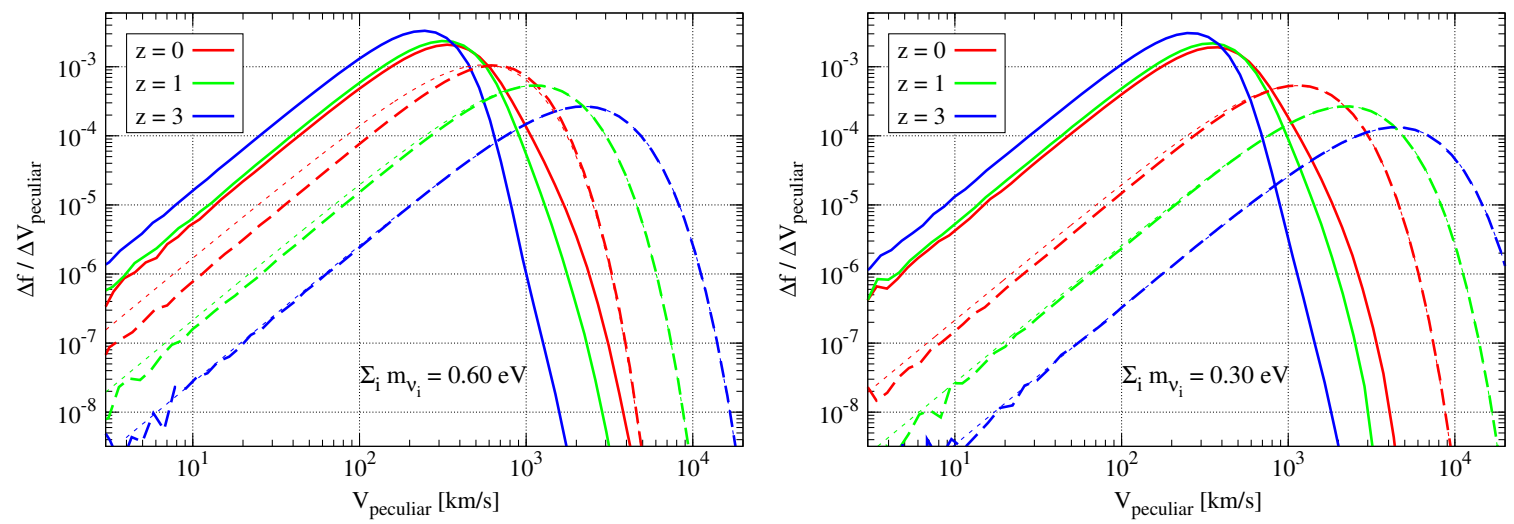

Figure 4. Fraction of particles with peculiar velocities between $V$ and $V+\triangle V$, per $\triangle V$, as a function of the peculiar velocity modulus. Solid lines show the results for the CDM particles at redshifts $z=0$ (red), $z=1$ (green) and $z=$ 3 (blue). Thick long-dashed lines represent the distribution of the peculiar velocities of the neutrino particles whereas thin short-dashed lines correspond to the unperturbed neutrino thermal velocity distribution at each redshift (Fermi-Dirac distribution).

velocities as times evolves as a consequence of the universe expansion. In order to study the impact on the neutrino velocity field of processes such as neutrino clustering within CDM haloes or neutrino evacuation from cosmological voids, we plot with thin short-dashed lines the unperturbed distribution for the neutrino peculiar velocities obtained from Eq. 1.1:

$$
\frac{d f}{d V_{\text {ther }}^{\nu}}(z)=\left(\frac{m_{\nu}}{1.803 c k_{B} T_{\nu}(z)}\right) \frac{x^{2}}{e^{x}+1},
$$

where $x=p_{\nu} /\left(k_{B} T_{\nu}(z)\right)$ and $f(V, z) d V$ is the fraction of neutrinos with thermal velocities between $V$ and $V+d V$. We have used the approximation $p_{\nu} \cong m_{\nu} V_{\text {ther }}^{\nu} / c$, with $V_{\text {ther }}^{\nu}$ the modulus of the neutrino thermal velocity, which is very accurate once neutrinos are non-relativistic. At redshifts $z>3$, the fully non-linear distribution of the modulus of the neutrino peculiar velocities is very well described by the unperturbed cosmic neutrino distribution of Eq. 3.1. However, at lower redshift, the peculiar velocities of some neutrinos are small enough to allow them to cluster within the gravitational potential wells of CDM halos or to evacuate the interior of cosmological voids. For that reason, the fraction of neutrinos with low velocities will be smaller in the fully non-linear regime than in the linear regime, since it is likely that those neutrinos will gain gravitational energy. Since the thermal velocities of relic neutrinos increase as their masses drop, the deviation of the neutrino velocity distribution from the unperturbed velocity distribution of Eq. 3.1 distribution becomes smaller for smaller neutrino masses. We find a constant suppression in the fraction of neutrino particles with low velocities with respect to the unperturbed distribution (Eq. 3.1). At redshift zero, the proportion of neutrinos with peculiar velocities smaller than $100 \mathrm{~km} / \mathrm{s}$ is a factor $\cong 2.08,1.72,1.33$, and 1.15 smaller than the FermiDirac distribution for neutrinos with $\Sigma_{i} m_{\nu_{i}}=0.60,0.45,0.30$, and $0.15 \mathrm{eV}$, respectively. We also find that the maximum of the actual distribution is shifted with respect to the unperturbed distribution. At $z=0$, the peak of the actual velocity distribution is located at $V \cong 640,805,1145,2220 \mathrm{~km} / \mathrm{s}$ for neutrinos with $\Sigma_{i} m_{\nu_{i}}=0.60,0.45,0.30,0.15 \mathrm{eV}$, respectively, whereas the peak in the unperturbed distribution is placed at $V \cong 560,745,1120,2235 \mathrm{~km} / \mathrm{s}$, respectively. The results for the simulation with $\Sigma_{i} m_{\nu_{i}}=0.05 \mathrm{eV}$ are very close to those from the simulation with $\Sigma_{i} m_{\nu_{i}}=0.15 \mathrm{eV}$. We shall see on Sec. 3.4.2 that the neutrino dynamics (of the massive species) in both simulations are basically the same. The high velocity tail is, in all cases, very well reproduced by the unperturbed neutrino velocity distribution.

We have investigated in detail the behavior of the neutrinos with low thermal velocities over redshift. In the left panel of Fig. 5 we plot with a solid orange line the number of neutrinos with $\Sigma_{i} m_{\nu_{i}}=0.60 \mathrm{eV}$ and with momentum between $p$ and $p+\triangle p$, per $\triangle p$ and per cubic meter, at $z=99$, as a function of the neutrino thermal velocity modulus. At this redshift, we take different velocity intervals that we show with vertical lines in the same panel. For a particular velocity interval, at $z=99$, we find all the neutrino particles whose thermal 

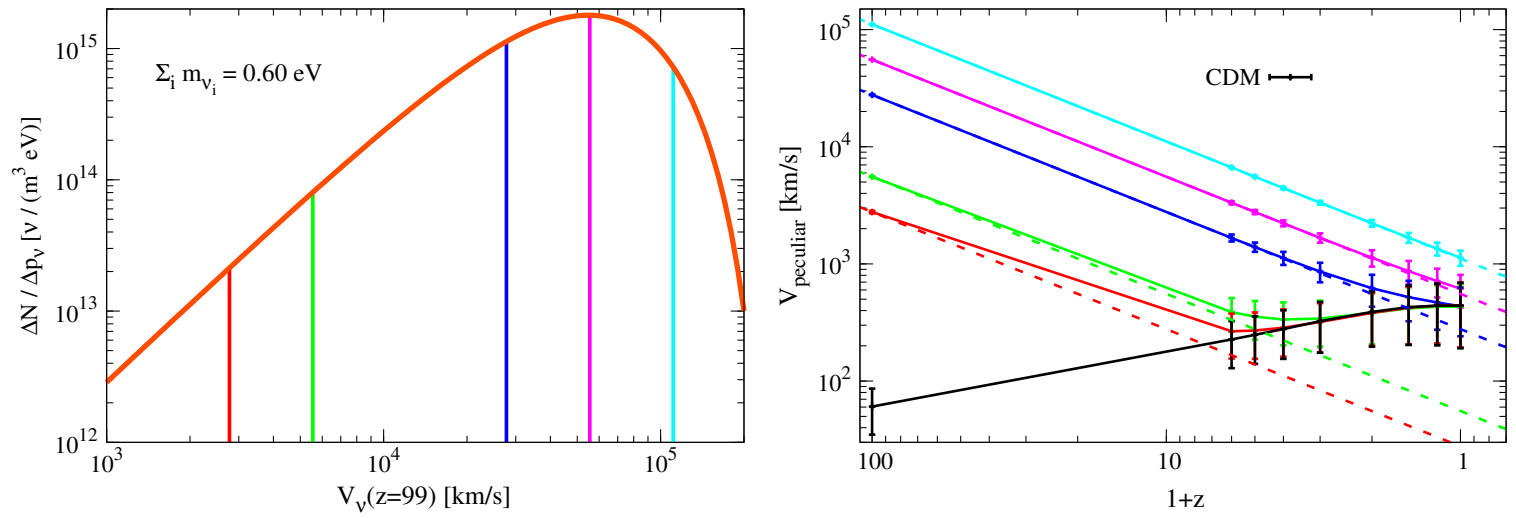

Figure 5. For neutrinos with $\Sigma_{i} m_{\nu_{i}}=0.60 \mathrm{eV}$, we show in the left panel with a solid orange line, the number density of neutrinos per unit of momentum as a function of the neutrino thermal velocity at $z=99$. As a function of redshift, the points and error bars in the right panel represent the mean and the velocity dispersion of the neutrino particles, whose thermal velocities at $z=99$ lie within the narrow velocity intervals that we show with vertical lines on the left panel (see text for details). The dashed lines in the right panel show the unperturbed evolution of the neutrino particles whose thermal velocities at $z=99$ lie within the different velocity intervals of the left panel. The black line represents the mean velocity and the velocity dispersion of a random but representative set of CDM particles as a function of the redshift.

velocities lie within it and store their IDs (integer numbers used to identify particles along the simulation). We then use those IDs to find the neutrino particles whose peculiar velocities belonged to a particular velocity interval at $z=99$, and compute their peculiar velocities at a posterior time. We perform this procedure at different redshifts and in the right panel of Fig. 5, we plot the mean and the velocity dispersion of the neutrinos whose thermal velocities lie within the different velocity intervals at $z=99$. The color of the points and curves are used to distinguish the different velocity intervals at $z=99$. The dashed lines show the unperturbed time evolution of the neutrinos, whose velocities drop as $1 /(1+z)$. We have also taken a representative set of CDM particles at $z=99$ and followed their evolution along time (black solid line in the right panel of Fig. 5). By representative we mean that this set is small, in comparison with the total number of CDM particles in the simulation, but large enough to make sure that the quantities we compute are converged. Whereas neutrinos with initial large thermal velocities follow very well the unperturbed evolution (see lines in purple and cyan), neutrinos with lower velocities behave in a different way. Once neutrinos are cold enough, their mean velocity, velocity dispersion and velocity evolution become the same as the this from the CDM. The redshift at which neutrinos catch up the behavior of the CDM depends on their initial momentum as can be seen in the right panel of Fig. 5. We find that neutrinos with velocities $\sim 1 / 20$ and $\sim 1 / 10$ of the mean neutrino thermal velocity at $z=99$, start behaving as CDM at redshifts $z \sim 3$ and $z \sim 2$, respectively. We therefore conclude that the deviations in the neutrino peculiar velocity distribution from the unperturbed distribution of Eq. 1.1 are mainly driven by neutrinos with low momentum, which at some redshift start behaving similarly to CDM.

\subsection{The halo mass function}

We study the effects of massive neutrinos on the halo mass function (MF) and compare with the ST (ShethTormen) [48, 49] prediction. This has been already presented in [41, 55]. In [41] authors limited their study at $z=0$. In [55], the impact of the neutrino masses on the halo mass function was studied at different redshifts but the simulations were performed with the grid implementation (see [11]), which can not capture fully the non-linear neutrino regime at small scales. Here we aim at improving over previous studies by computing the impact of neutrino masses on the halo mass function at different redshifts, using the particle implementation for the neutrino particles and for a very wide range of halo masses.

From the N-body simulations we identify the CDM haloes and extract their properties by applying the SUBFIND algorithm [47]. A CDM halo corresponds to a group identified by SUBFIND, where its virial radius is defined as $M=\frac{4 \pi}{3} \triangle_{\text {vir }} \bar{\rho}_{\mathrm{c}}(z) R_{\text {vir }}^{3}$ with $\triangle_{\mathrm{vir}}$ being the value of the mean overdensity at the time of virialisation as predicted by the top-hat collapse model [54], $\triangle_{\text {vir }}=18 \pi^{2}+82 x-39 x^{2}$, where $x=$ 

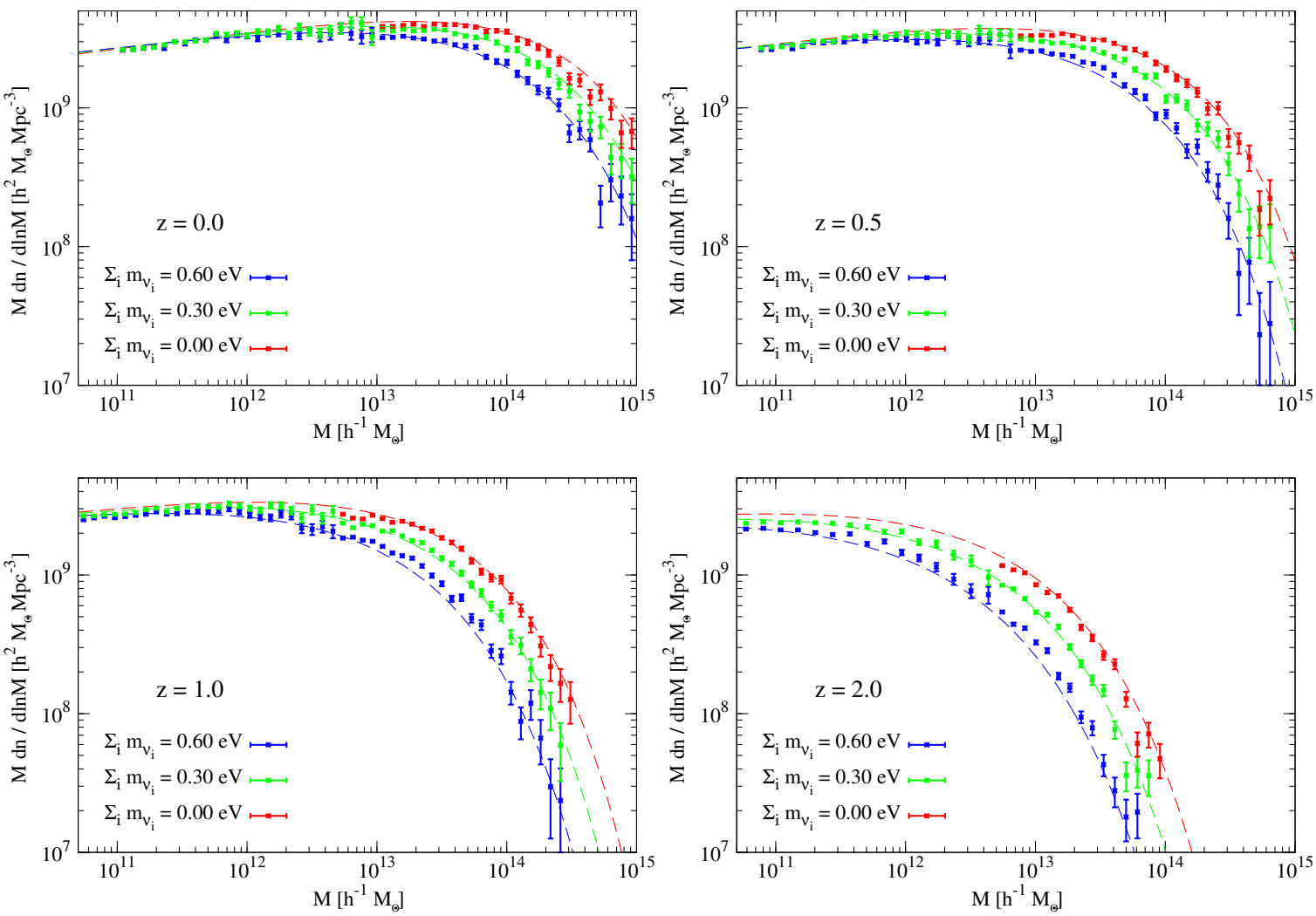

Figure 6. Halo mass function for different cosmologies with different $\Omega_{\nu}$ satisfying $\Omega_{\mathrm{CDM}}+\Omega_{b}+\Omega_{\nu}=0.2708$. In all the models the amplitude of the power spectra is fixed to the same value on large scales (thereby the simulations have different $\sigma_{8}$ values). The mass functions are shown for $z=0,0.5,1,2$ in the top left, top right, bottom left and bottom right panels, respectively, for two different total neutrino masses of $0.30 \mathrm{eV}$ (green points) and $0.60 \mathrm{eV}$ (blue points). The massless neutrino case is shown with red points. The theoretical modified Sheth-Tormen predictions are shown as dashed curves.

$-\Omega_{\Lambda} /\left(\Omega_{\mathrm{m}}(1+z)^{3}+\Omega_{\Lambda}\right)$. The linear matter power spectrum, that impacts on the ST formula through $\sigma(M)$, is computed using CAMB [53] taking the same cosmological parameters of the N-body simulations.

The MFs from N-body simulations and the ST fits are plotted on Fig. 6 and the error bars correspond to the statistical Poisson noise. The mass functions are extracted from both the low resolution simulations (L60, L30 and L0) and the high resolution simulations (S60 and S30) at different redshifts: $z=0,0.5,1$ and 2. The agreement between N-body simulations and the ST formula is reasonably good as already found by [41, 55]. However, for neutrinos with $\Sigma_{i} m_{\nu_{i}}=0.60 \mathrm{eV}$, we find a significantly larger halo abundance in the N-body simulations than in the ST prediction. Those deviations are larger for haloes of $\sim 10^{13} h^{-1} \mathbf{M}_{\odot}$, independently of redshift. The ST profiles are obtained by using the value of $\Omega_{\mathrm{CDM}}+\Omega_{\mathrm{b}}$ as $\Omega_{\mathrm{M}}$ instead of $\Omega_{\mathrm{CDM}}+\Omega_{\mathrm{b}}+\Omega_{\nu}$. This point was discussed in detail in [41]. The reason why $\Omega_{\mathrm{m}}$ should be computed without including the neutrino component is because the clustering of neutrinos within CDM haloes is very small, as we will see in the following Section. Thus, it is a very good approximation to assume that neutrinos, at least for the masses considered here, do not participate to the clustering process and neglect their contribution to the $\Omega_{\mathrm{M}}$ value.

\subsection{The neutrino halo}

In this Section we compute the neutrino density profiles around CDM haloes. Further, we provide the reader with a fitting function that reproduces the neutrino profiles with high accuracy over a wide range of radii. 
The average velocity dispersion of CDM haloes is well described by the formula [56]:

$$
\sigma_{D M}(M, z)=\sigma_{D M, 15}\left[\frac{h(z) M_{200}}{10^{15} \mathrm{M}_{\odot}}\right]^{\alpha},
$$

where $h(z)=H(z) /\left(100 \mathrm{~km} \mathrm{~s}^{-1} \mathrm{Mpc}^{-1}\right), \mathrm{M}_{200}$ is the mass within the virial radius (in this case defined as the radius at which the mean density is 200 times larger than the critical density), $\sigma_{D M, 15}$ and $\alpha$ are constants with values $\sim 1080 \mathrm{~km} / \mathrm{s}$ and $\sim 0.336$ respectively. Thus, at redshift $z=0$, CDM haloes have typical velocity dispersions ranging from $\sim 100 \mathrm{~km} / \mathrm{s}$, for halo masses of $10^{12} h^{-1} \mathrm{M}_{\odot}$, to $\sim 1000 \mathrm{~km} / \mathrm{s}$ for halo masses of $\sim 10^{15} h^{-1} \mathrm{M}_{\odot}$. For a fixed CDM halo mass, the velocity dispersion grows with redshift as $h(z)^{\alpha}$.

On the other hand, the mean thermal velocity of neutrinos is equal to $160(1+z)\left(\mathrm{eV} / m_{\nu}\right) \mathrm{km} / \mathrm{s}$, while their velocity dispersion reads:

$$
\sigma_{\nu}=c\left(\frac{\mathrm{eV}}{m_{\nu}}\right) \sqrt{\frac{\int_{0}^{\infty} n_{\nu}(p, z)\left(p-\bar{p}_{\nu}(z)^{2}\right) d p}{\int_{0}^{\infty} n_{\nu}(p, z) d p}},
$$

which implies $\sigma_{\nu} \sim 87(1+z)\left(\frac{\mathrm{eV}}{m_{\nu}}\right) \mathrm{km} / \mathrm{s}$. Therefore, we would expect relic neutrinos to cluster within the gravitational potential wells of CDM haloes, at least for the most massive neutrinos. This clustering will be larger for higher neutrino masses, and we would expect that it starts at low redshift, since at high redshift the low velocity dispersion of CDM haloes and the large neutrino thermal velocities will prevent it.

The clustering of neutrinos within the gravitational potential wells of CDM haloes has already been studied using semi-analytic models [18-20, 22] and N-body simulations [41]. In [41], it was demonstrated that the agreement between both methods is fairly good. The differences found were likely due to the simplified assumptions used in [19] to compute the neutrinos clustering, e.g. the fact that the gravitational potential wells of CDM were assumed not to change with time. A more realistic calculation can be found in [20]. Here we compute the neutrino density profiles within CDM haloes using a different set of N-body simulations, with slightly different values of the cosmological parameters and with the improved code GADGET-3.

The CDM haloes are identified from the N-body simulations as groups by the algorithm SUBFIND (see Sec. 3.3 for further details). We focus our study on the clustering of neutrinos within isolated CDM haloes. Our choice of focusing on isolated CDM haloes is deliberate: the clustering of neutrinos is very sensitive to the CDM distribution on large scales, therefore, by focusing on isolated CDM haloes we will obtain results that will have less dispersion than in the general case. In Appendix A, we show how the neutrino profiles change when we compute the clustering of neutrinos within non-isolated CDM haloes.

We define a CDM halo as isolated if no more massive CDM haloes are situated at a distance less than 10 times its virial radius If the former condition is not satisfied, then the CDM halo is non-isolated. We study the clustering of relic neutrinos within CDM haloes of different masses at $z=0: 10^{11}, 10^{12}, 10^{13}$ and $10^{14}$ $h^{-1} \mathrm{M}_{\odot}$. For a given mass of the host CDM halo, we create a halo catalog consisting of all haloes whose virial masses differ from this value by less than $5 \%$. This tolerance is chosen in order to increase the number of haloes over which we compute quantities, i.e. improve the statistical significance of the results, while keeping it low enough to avoid selecting haloes with very different properties (such as the value of their virial radius). For a given mass of the host CDM halo we compute the density profile for the CDM component for all of its halo members, at $z=0$, and in Fig. 7 we show the mean density profiles for the different halo catalogs. The profiles shown in the figure have been extracted from the simulation L60, i.e. for the cosmology with $\Sigma_{i} m_{\nu_{i}}=0.60 \mathrm{eV}$. We find that the average CDM density profiles are very well fitted by the Navarro-Frenk-White (NFW) profile [57] $\rho_{N F W}(r)=\frac{\rho_{s}}{\left(r / r_{s}\right)\left(1+r / r_{s}\right)^{2}}$, up to the virial radius. We have also computed the average CDM density profiles for the cosmologies with $\Sigma_{i} m_{\nu_{i}}=0.45,0.30,0.15$ and $0.00 \mathrm{eV}$ and it turns out that, in all cases, the mean density profiles are almost identical. However, we find that the halo concentration, $c=R_{v i r} / r_{s}$, slightly decreases with the neutrino masses. This behavior was also found by [41] and, as they discussed, it is likely due to the fact that massive haloes form at later times in cosmologies with massive neutrinos, as we have seen from the halo mass function in Sec. 3.3.

We repeat the same procedure for the neutrino component and in Fig. 8 we show the average neutrino density profiles at $z=0$ normalized to the neutrino background density. The error bars represent the dispersion in the average neutrino overdensity profile, that we compute in the following way: for a given halo catalog, 


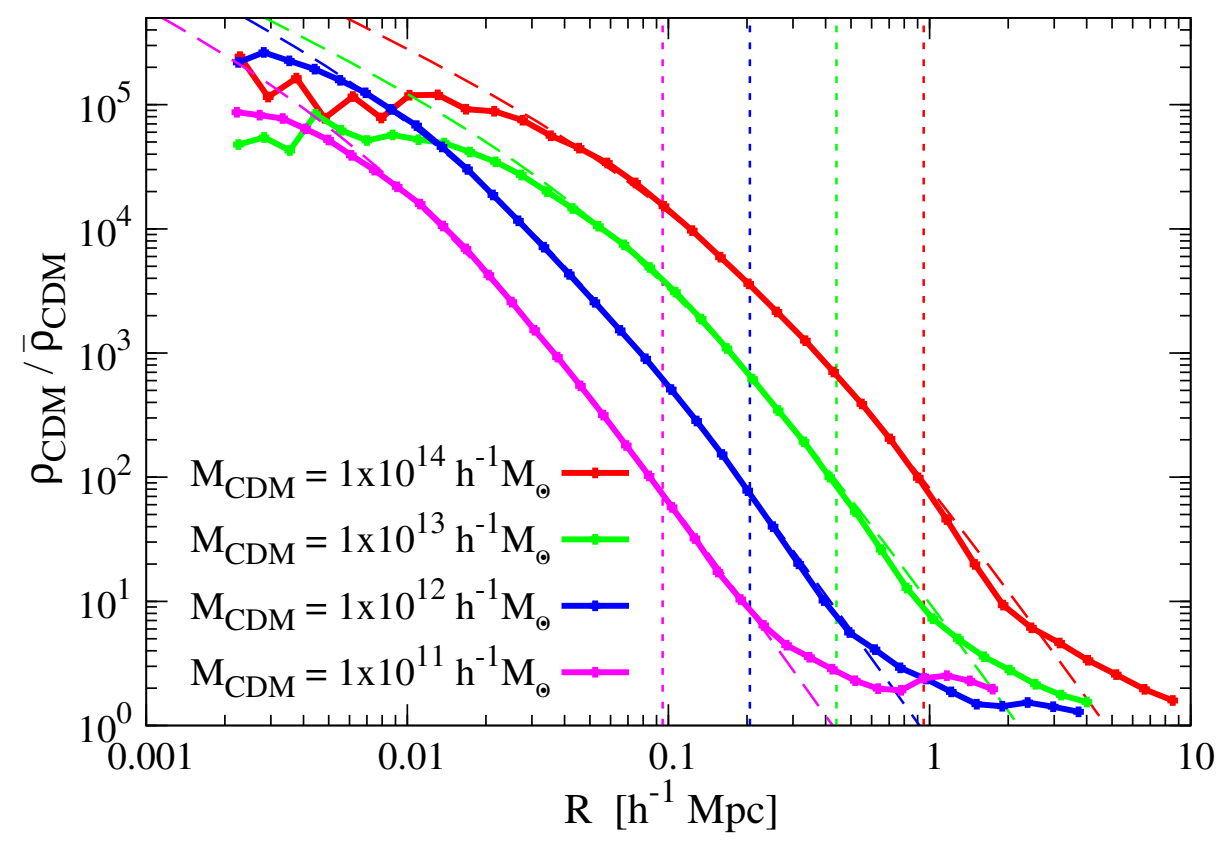

Figure 7. Average CDM density profiles at $z=0$. The points show the mean density profile for the CDM component as a function of radius for four different CDM halo masses at redshift zero: $1 \times 10^{14}$ (red), $1 \times 10^{13}$ (green), $1 \times 10^{12}$ (blue) and $1 \times 10^{11}$ (purple) $h^{-1} \mathrm{M}_{\odot}$ (for each mass we select all CDM haloes within a mass bin of width $5 \%$ ). The vertical lines correspond to the values of the virial radius for the different haloes, whereas the dashed lines show the NFW profile that best fits each profile.

we compute the mean overdensity profile and the dispersion around it. We then assume that, for a given radius bin, the overdensity values are distributed following a Gaussian distribution, and thus, the dispersion in the mean overdensity profile will be given by $\sigma_{\nu}^{\text {profile }}(r) / \sqrt{N}$, where $\mathrm{N}$ is the number of halos in the catalog and $\sigma_{\nu}^{\text {profile }}(r)$ is the dispersion around the mean overdensity profile. The neutrino density profiles are computed for each host CDM halo mass extracted from the simulations L60, L45, L30 and L15 for four different neutrino masses: $\Sigma_{i} m_{\nu_{i}}=0.60,0.45,0.30$ and $0.15 \mathrm{eV}$, respectively. We shall see in Sec. 3.4 .2 that the overdensity profiles for a cosmology with $\Sigma_{i} m_{\nu_{i}}=0.05 \mathrm{eV}$ would be equivalent to those obtained from L15. The noisy behavior that arises at small scales is due to the finite number of particles and to the finite number of CDM haloes. Therefore, on small scales, the dispersion in the average neutrino density profiles is relatively large, pointing out our resolution limits.

For a given sum of the neutrino masses, the clustering of relic neutrinos increases with the masses of their host CDM haloes, since more massive haloes have larger and deeper gravitational potential wells than less massive ones. For a fixed mass of the host CDM halo, the higher the neutrino masses the larger the neutrino clustering will be: this is because the proportion of neutrinos with low or moderate peculiar velocities, the ones subjected to clustering, increases with the neutrino masses. In fact, due to their large thermal velocities, neutrinos are not able to cluster on small scales: thus, the neutrino profiles are not cuspy but they exhibit a relatively large core.

For Milky way size haloes $\left(\sim 10^{12} h^{-1} \mathrm{M}_{\odot}\right)$ our numerical resolution do not allow us to explore the inner regions of the neutrino density profile. We therefore conclude that the relic neutrino overdensity at the solar radius has to be larger than $\sim 40 \%$, with respect to this of the background, for neutrinos with $\Sigma_{i} m_{\nu_{i}}=0.60$ $\mathrm{eV}$ and above $\sim 10 \%$ for neutrinos with $\Sigma_{i} m_{\nu_{i}}=0.30 \mathrm{eV}$.

Although the values of the cosmological parameters in [41] are different to ours, we find that our results are in good agreement with their results. We also find a reasonable agreement with the neutrino overdensity profiles computed through the $\mathrm{N}$-one-body method described in $[19,20]$.

We also investigate the dependence of the neutrino density profiles with redshift for a fixed mass of the 

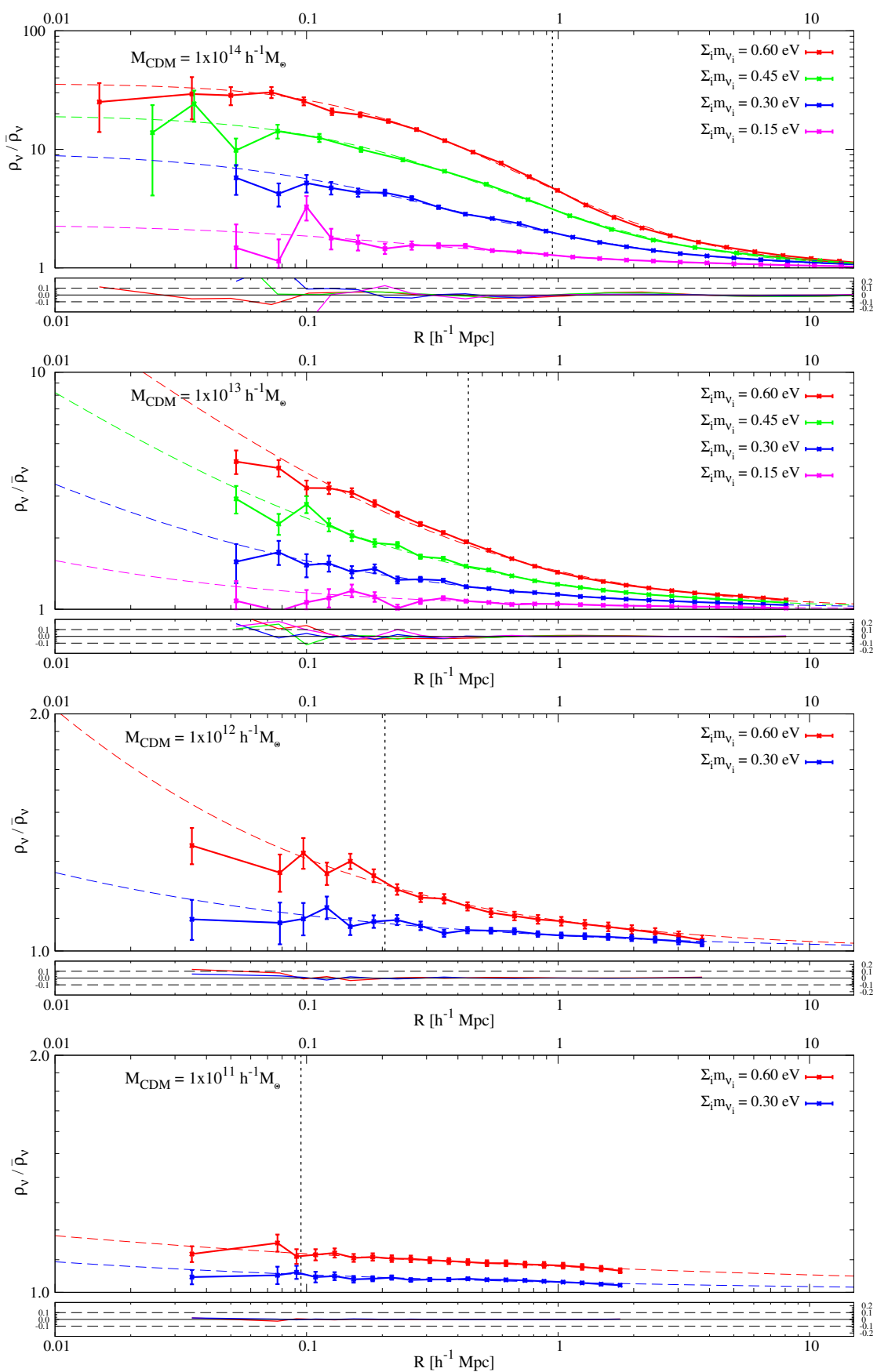

Figure 8. Mean neutrino overdensity profiles for different neutrino masses and for different masses of their host CDM haloes. Each panel shows the average neutrino density profiles at $z=0$, normalized to the mean neutrino density, that arise due to the clustering of neutrinos within CDM haloes of masses $1 \times 10^{14} h^{-1} M_{\odot}$ (first panel), $1 \times 10^{13} h^{-1} M_{\odot}$ (second panel), $5 \times 10^{12} h^{-1} M_{\odot}$ (third panel), $1 \times 10^{11} h^{-1} M_{\odot}$ (fourth panel) at $z=0$. The error bars show the dispersion of the mean overdensity profile. The profiles are computed for cosmologies with $\sum_{i} m_{\nu_{i}}=0.60 \mathrm{eV}$ (red), $0.45 \mathrm{eV}$ (green), $0.30 \mathrm{eV}$ (blue) and $0.15 \mathrm{eV}$ (purple). Dashed lines represent the profiles of Eq. 3.4 that best fit the computed average neutrino overdensity profiles. On the bottom of each panel we plot the relative difference between the profiles and the fitting formula. The value of the virial radius for the different host CDM halo masses is shown in each panel with a vertical line. 

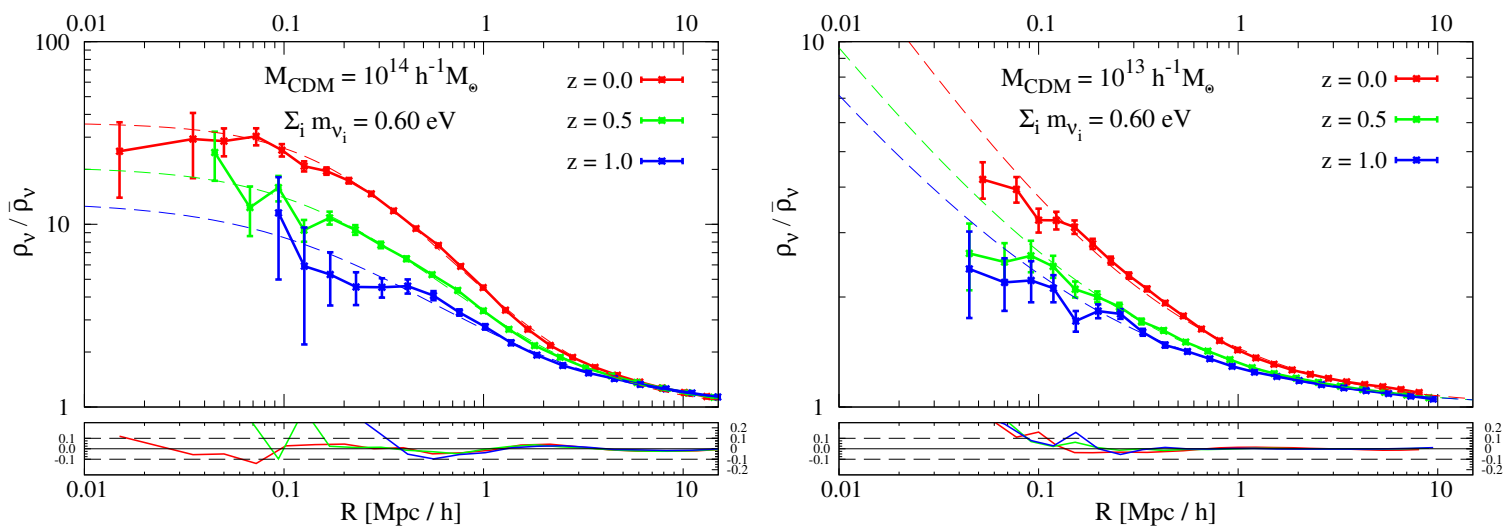

Figure 9. Dependence of the neutrino clustering with redshift for a fixed mass of the CDM halo. We plot the neutrino overdensity profiles at redshifts $z=0$ (red), $z=0.5$ (green) and $z=1$ (blue) within CDM haloes that have masses equal to $10^{14} h^{-1} \mathrm{M}_{\odot}$ at $z=0$ (red), $z=0.5$ (green) and $z=1$ (blue). The right panel shows the same quantities but for CDM haloes of masses $10^{13} h^{-1} \mathrm{M}_{\odot}$. In both panels the density profiles refer to the $\Sigma_{i} m_{\nu_{i}}=0.60 \mathrm{eV}$ case.

host CDM halo. That is done by computing the neutrino density profiles within CDM haloes that have the same virial mass at different redshifts. The results are shown in Fig. 9 for two different masses of the host CDM halo and for the $\Sigma_{i} m_{\nu_{i}}=0.60 \mathrm{eV}$ case. In the left panel, the red line represents the average neutrino density profile at $z=0$, computed for host CDM haloes that have a virial mass equal to $10^{14} h^{-1} \mathrm{M}_{\odot}$ at $z=0$; the green (blue) line corresponds to the average neutrino density profile at $z=0.5(z=1)$, computed within CDM haloes that have a virial mass equal to $10^{14} h^{-1} \mathrm{M}_{\odot}$ at $z=0.5(z=1)$; the $x$-axis represents the distance to the halo center in comoving units. The right panel shows the same for host CDM haloes with masses equal to $10^{13} h^{-1} \mathbf{M}_{\odot}$.

Although CDM haloes have the same masses, the thermal velocities of neutrinos are larger at high redshift $^{8}$. As a consequence, the clustering of neutrinos becomes smaller as redshift increases.

\subsubsection{Fitting function}

We find that the average neutrino overdensity profiles are well described by the following equation:

$$
\delta_{\nu}(r)=\frac{\rho_{\nu}(r)-\bar{\rho}_{\nu}}{\bar{\rho}_{\nu}}=\frac{\rho_{c}}{1+\left(r / r_{c}\right)^{\alpha}}
$$

over a wide range of radii. The physical meaning of the parameters in the profile 3.4 is very simple: $r_{\mathrm{c}}$ and $\rho_{\mathrm{c}}$ represent the length and the overdensity of the core in the overdensity profile of the neutrino halo while $\alpha$ is a parameter that controls how fast the overdensity profile falls on large radii. However, for CDM halo masses below $\sim 10^{13.5} h^{-1} \mathrm{M}_{\odot}$, the resolution in our N-body simulations is not large enough to properly resolve the core in the neutrino density profiles ${ }^{9}$. This gives rise to a degeneracy between the parameters $\rho_{\mathrm{c}}$ and $r_{\mathrm{c}}$. We find that a simple profile of the form:

$$
\delta_{\nu}(r)=\kappa / r^{\alpha}
$$

reproduces the outskirts of the computed neutrino density profiles very well. Note that on distances much larger than the core radius, $r \gg r_{\mathrm{c}}$, the profile 3.4 reduces to 3.5 , with $\kappa=\rho_{\mathrm{c}} r_{\mathrm{c}}^{\alpha}$. For a given average neutrino overdensity profile, the values of the parameters $\vec{p}=\left(\rho_{\mathrm{c}}, r_{\mathrm{c}}, \alpha\right)(\vec{p}=(\kappa, \alpha)$ for CDM halos masses below $\left.\sim 10^{13.5} h^{-1} \mathrm{M}_{\odot}\right)$ are those that minimize the quantity:

$$
\chi^{2}=\sum_{i}\left[\frac{\delta_{\nu}^{i}-\delta_{v}\left(r_{i}, \vec{p}\right)}{\sigma_{\nu}^{i}}\right]^{2},
$$

\footnotetext{
${ }^{8}$ Note also that the CDM halo velocity dispersion grows with redshift $\left(\propto h(z)^{\alpha}\right)$ more slowly than the mean and velocity dispersion of relic neutrinos $(\propto(1+z))$

${ }^{9}$ We note that a core is always expected because of the Tremaine-Gunn bound [58]
} 

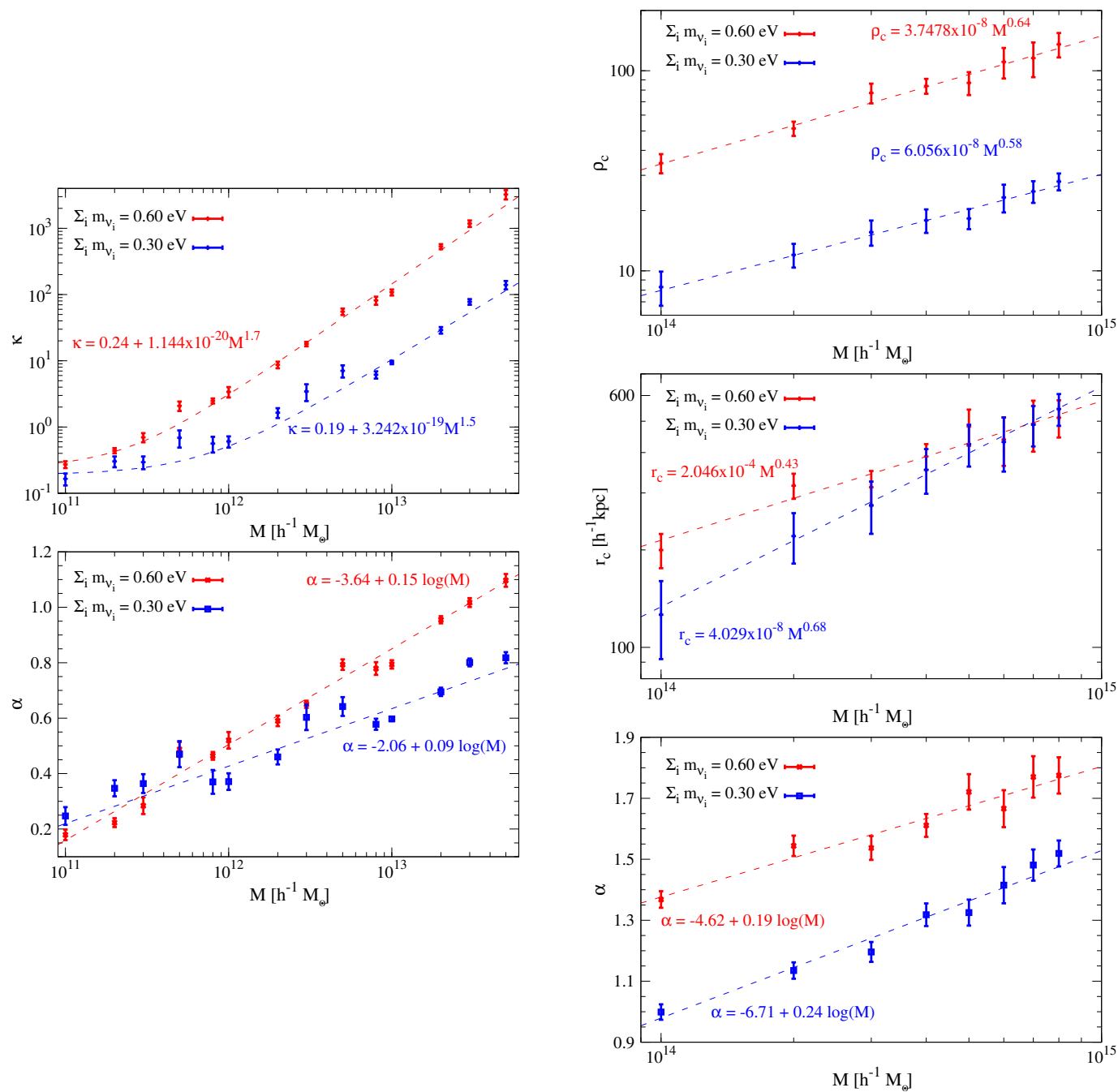

Figure 10. Derived parameters from the fitting function used in order to investigate the neutrino density profile. The panels on the right show the dependence of the parameters of the profile of Eq. 3.4 with the neutrino masses and the masses of their host CDM halo. For lighter CDM halos, our N-body resolution do not allow us to resolve properly the parameters $\rho_{\mathrm{c}}$ and $r_{\mathrm{c}}$ (see text for details). The panels on the left show the dependence of the parameters for the simpler profile of Eq. 3.5 with the neutrino masses and CDM halo masses.

where $\delta_{\nu}^{i}$ and $\sigma_{\nu}^{i}$ are the values of the average neutrino overdensity and overdensity dispersion at radius $r_{\mathrm{i}}$, respectively. The fitting formula, with the value of their parameters extracted as above, are plotted as dashed lines in Fig. 8. In the bottom part of the panels in Fig. 8 we plot the relative difference between the fitting formula and the average density profiles.

The outskirts of the neutrino haloes are very well reproduced by the fitting formula for all CDM halo masses, although it works best for lower CDM halo masses. At large radii, the fitting formula is more accurate for small values of the neutrino masses. When using the power-law profile (Eq. 3.5), important discrepancies between the fitting formula and the neutrino density profiles take place on small scales. This happens because the fitting formula of Eq. 3.5 is cuspy, whereas the neutrino density profiles must exhibit a core (see for example [19]). We note that the former formula will eventually violate the Tremaine-Gunn bound [58], and for that reason, the extrapolated values of the fitting formula should be taken with caution.

The values of the fit parameters depend on three quantities: the mass of the host CDM halo, redshift 
and the masses of the neutrinos. We investigate the dependence of the fitting formula parameters on neutrino masses and on the mass of the host CDM halo. In Fig. 10 we plot the values of the fitting profile parameters, extracted as explained above, as a function of the mass of the CDM halo that hosts the neutrino halo for two different neutrino masses. Both the CDM halo masses and the average neutrino profiles from which the parameters are extracted are at $z=0$. The red points represent the values of the parameters for neutrinos of masses $\Sigma_{i} m_{\nu_{i}}=0.60 \mathrm{eV}$, while the blue points are for neutrinos of masses $\Sigma_{i} m_{\nu_{i}}=0.30 \mathrm{eV}$. The error bars correspond to the $1 \sigma$ errors on the value of the parameters, while the dashed lines represent a simple fitting formula that fits the values of the parameters reasonable well (see appendix B).

We check whether the fitting formula of Eq. 3.4 (3.5 when the core is not properly resolved) can reproduce the average density profiles of neutrino haloes at $z>0$. The fitting profiles, whose parameter values are extracted as above, are shown with dashed lines in Fig. 9 for different masses of the host CDM halo at different redshifts. The relative difference between the density profile points and the fitting formula is shown at the bottom of each panel on that figure: the fitting profile reproduces very well the outskirts of the computed profiles at all redshifts. The inner region is better described when the neutrino clustering is large enough to properly resolve the core in the density profile.

\subsubsection{Convergence tests}

We now investigate whether our results are numerically stable with respect to: number of neutrino particles, size of the simulation box and starting redshift of the simulation. In the left panel of Fig. 11, we show the results of computing the mean neutrino density profiles at $z=0$, within isolated CDM haloes of masses $10^{14}$ $h^{-1} M_{\odot}$ at redshift $z=0$, for different neutrino masses, by using several simulations with the same box size but different number of neutrino particles. In particular, we compare the results from the simulations L60 and L45 to those obtained from LL60 and L45. We find that by increasing the number of neutrino particles by a factor eight, keeping fixed the size of the simulation box, the density profiles vary less than $10 \%$ for radii larger than $\sim 200 h^{-1} \mathrm{kpc}$. We therefore conclude that our results are already converged for those radii even for the low resolution simulations. At smaller radii, it turns out that the results are more stable the more massive the neutrinos are. This is a consequence of the discrete sampling of the neutrino phase-space: the lower the neutrino masses the higher the neutrino phase-space distribution has to be sampled to resolve the small scale features in the neutrino density profiles.

In the left panel of Fig. 11 we also show the neutrino overdensity profiles extracted from the simulations L15 and LL5. Whereas the simulation L15 contains three degenerate species of neutrinos having each of them a mass equal to $0.05 \mathrm{eV}$, the simulation LL5 consists of two massless neutrino species and one massive species with a total mass equal to $0.05 \mathrm{eV}$. Although the matter power spectrum is different in both simulations (see table 1), the neutrino momentum distribution and the CDM halo velocity dispersion are basically identical. For that reason, we find that the results are the same in both cases, being converged at the $10 \%$ level for radii larger than $200 \mathrm{~h}^{-1} \mathrm{kpc}$. We emphasize that this happens because we are considering the neutrino overdensity, a quantity which is insensitive to the number of degenerate species while the unnormalized neutrino density profile is a factor three larger in L15 with respect to LL5.

We repeat this analysis at different redshifts. We find that for CDM haloes of masses $10^{14} h^{-1} M_{\odot}$, the relative differences between the profiles computed from the low and from the high resolution simulation remain below $10 \%$ for radii larger than $400 h^{-1} \mathrm{kpc}$ at redshift $z=0.5$, whereas at redshift $z=1$ differences become larger than $10 \%$ for radii smaller than $700 h^{-1} \mathrm{kpc}$. At this redshift, the relative difference between the models with $\Sigma_{i} m_{\nu_{i}}=0.05 \mathrm{eV}$ and $\Sigma_{i} m_{\nu_{i}}=0.15 \mathrm{eV}$ keeps below $10 \%$ for radii larger than $400 \mathrm{~h}^{-1} \mathrm{kpc}$. The reason why differences become larger for larger neutrino masses is because at this redshift there are few CDM haloes with masses equal to $10^{14} h^{-1} M_{\odot}$, and therefore, the computed profiles are prone to the discreteness in the neutrino phase-space and to the cosmic variance.

We also study the dependence of the average neutrino density profiles on the size of the cosmological box and on the starting redshift of the simulation. We check this by running a N-body simulation with a box size of $1000 h^{-1} \mathrm{Mpc}, 512^{3} \mathrm{CDM}$ particles and $1024^{3}$ neutrino particles. The starting redshift is $z=19$, in contrast with our default choice of $z=99$. The simulation corresponds to the cosmological model with $\Sigma_{i} m_{\nu_{i}}=0.60$

$\mathrm{eV}$ and is listed on the table 1 as H60. In the right panel of Fig. 11 we show with dashed lines the average neutrino density profiles at $z=0$, for different masses of their host CDM haloes, extracted from the simulation H60. We compare those density profiles with those obtained from the simulation L60 (solid lines) and find that 

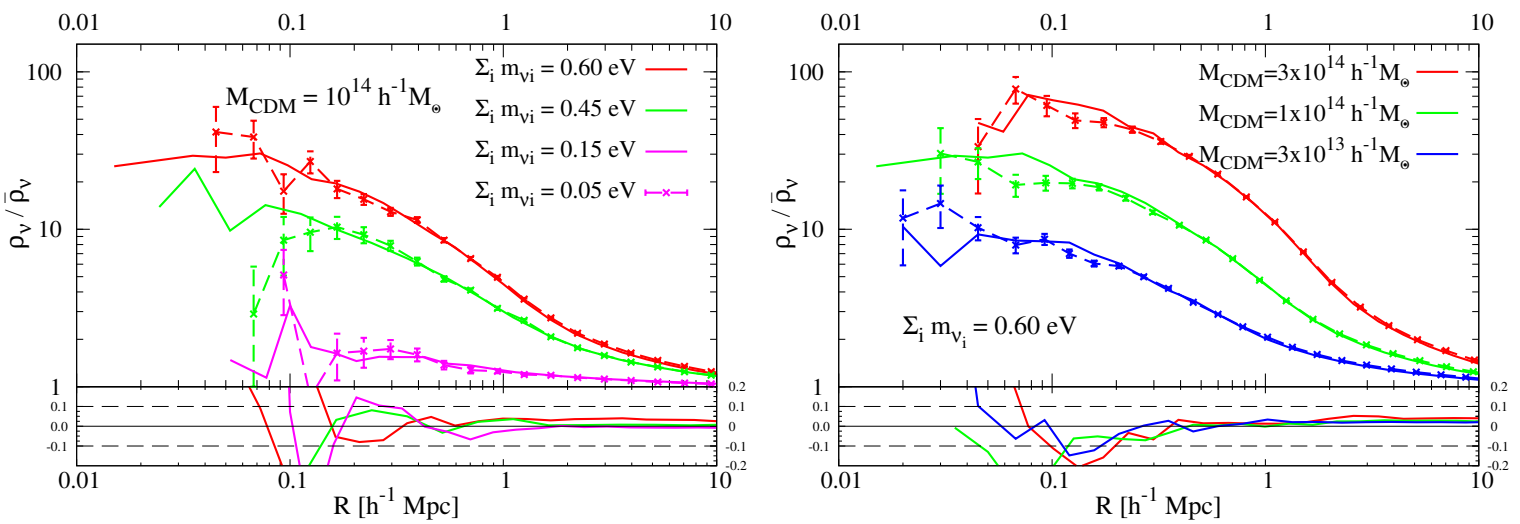

Figure 11. Left panel: Dependence of the neutrino overdensity profiles on the number of neutrino particles for a box of fixed size. The figure shows the mean neutrino density profiles for isolated CDM haloes of masses $10^{14} h^{-1} M_{\odot}$, at $z=0$, extracted from the simulations L60, L45, L15 (solid lines) and LL60, LL45, LL5 (dashed lines). In the bottom panel, we plot the relative difference between the low-resolution and the high-resolution profiles. Right panel: dependence of the neutrino overdensity profiles with the simulation box size and starting redshift. The solid lines show the mean neutrino density profile, at $z=0$, for different masses of their isolated host CDM haloes at $z=0: 3 \times 10^{14}$ (red), $1 \times 10^{14}$ (green) and $3 \times 10^{14} h^{-1} M_{\odot}$ (blue) extracted from the simulation L60. The dashed lines represent the same quantities but as extracted from the simulation H60. In the bottom panel, we plot the relative difference between the profiles obtained from H60 and those from L60. The error bars show the dispersion of the average neutrino overdensity profile for the simulations with lower resolution.
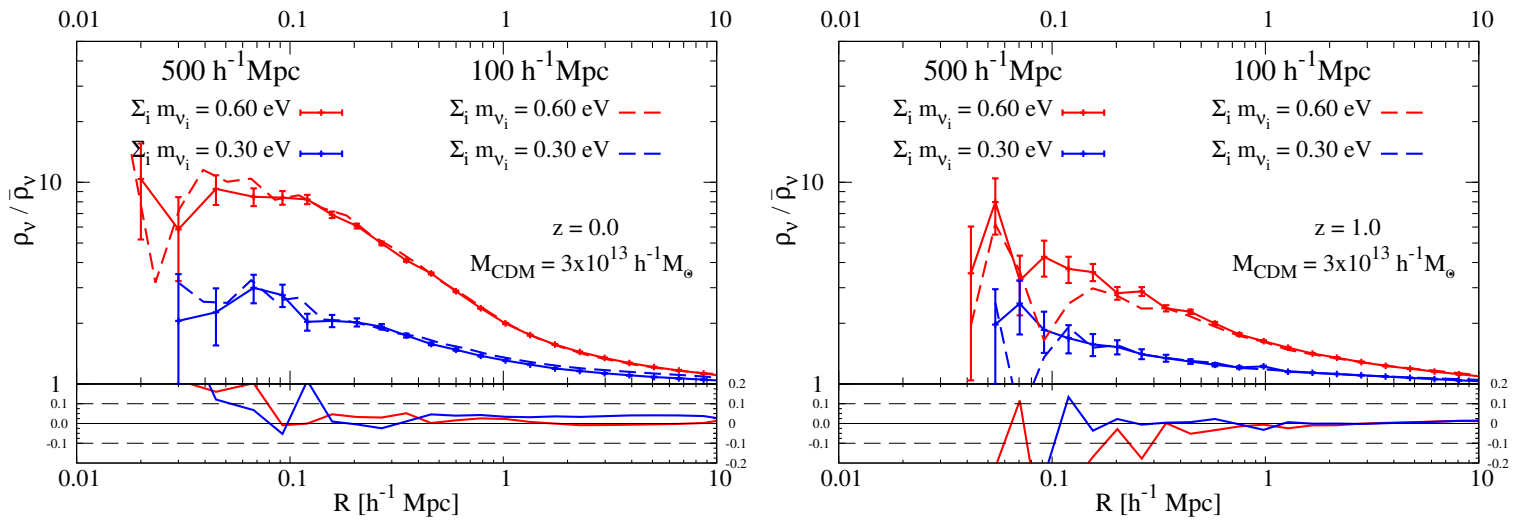

Figure 12. Comparison between the results of the simulations L60 and 30 (solid lines) and the results of the simulations S60 and S30 (dashed lines). For neutrinos with $\Sigma_{i} m_{\nu_{i}}=0.60 \mathrm{eV}$ (red) and $\Sigma_{i} m_{\nu_{i}}=0.30 \mathrm{eV}$ (blue) we plot the average neutrino density profiles, within CDM haloes of masses equal to $3 \times 10^{13} h^{-1} \mathrm{M}_{\odot}$ at $z=0$ (left) and at $z=1$ (right), extracted from the the simulations L60, S60, L30 and S30. At the bottom we show the relative difference between the profiles. The error bars show the dispersion of the average neutrino overdensity profile for the simulations with lower resolution.

both profiles differ by less than $10 \%$ for radii larger than $200 h^{-1} \mathrm{kpc}$, for all CDM halo masses. It is worth noting that in the simulation $\mathrm{H} 60$, the resolution of the CDM and neutrino particles are a factor eight below that of the simulation L60. The fact that the profiles do not depend on the initial redshift of the simulation should not be surprising since we have already seen that neutrino clustering starts at very recent times ${ }^{10}$.

Finally, we compare the neutrino density profiles extracted from the low resolution N-body simulations L60 and L30 to those obtained from the high resolution simulations S60 and S30. Since the size of the box in the simulations S60 and S30 is five times smaller than that of L60 and L30, the comparison can only be

\footnotetext{
${ }^{10}$ However, the starting redshift of the simulation has to be high enough for 2LPT to properly describe the CDM evolution.
} 
performed for CDM haloes of masses $\sim 10^{13} h^{-1} \mathrm{M}_{\odot}$. In Fig. 12 we plot the average neutrino density profiles, at redshifts $z=0,1$ within $\mathrm{CDM}$ haloes of masses equal to $3 \times 10^{13} h^{-1} \mathrm{M}_{\odot}$ extracted from the high and low resolution simulations. We find a very good agreement between both simulations. For CDM haloes of masses $(1-2) \times 10^{13} h^{-1} \mathrm{M}_{\odot}$ we find some discrepancies in the average neutrino density profiles, arising as a consequence of slightly different average CDM density profiles. This is likely due to the relatively low number of CDM haloes with those masses in the high resolution simulations $(\sim 30)$, which produces a slightly biased result with respect to the average. We therefore conclude that the results from the low resolution simulations are converged for CDM halo masses larger than $1 \times 10^{13} h^{-1} \mathbf{M}_{\odot}$.

\subsection{Neutrino velocity distribution within CDM haloes}

In this Section we study the distribution of the neutrino peculiar velocities within isolated CDM haloes. In Sec. 3.2 we have seen that the distribution of neutrino peculiar velocities computed over the whole set of simulated boxes is reasonably well described by the unperturbed neutrino momentum distribution of Eq. 1.1. However, at low redshift, the proportion of neutrinos with low peculiar velocities is over-estimated by the unperturbed distribution. As we showed in Fig. 5, this happens because neutrino momenta can not keep decreasing their value as $\propto 1 /(1+z)$, since once neutrino velocities are low enough, they will behave in the same way as CDM particles do.

By kinematical considerations, the proportion of neutrinos with low velocities within CDM haloes has to be small. On the other hand, we have seen that neutrino haloes are substantially more extended than their CDM counterparts, since typical neutrino peculiar velocities are larger than those of CDM.

We compute the distribution of the neutrino peculiar velocities within CDM haloes of different masses at $z=0$ by considering the distribution of neutrino momenta within the CDM halo virial radius. As in the case of the neutrino density profiles, for a given mass of the host CDM halo, we create a halo catalog by selecting all isolated CDM haloes whose masses differ of it by less than a 5\%. For each CDM halo belonging to a given catalog, we take all neutrino particles that lie within the CDM halo virial radius and compute the fraction of neutrinos in velocity bins. By fraction we mean the number of particles within a velocity interval over the total number of particles within the CDM halo virial radius. For a given sum of the neutrino masses, the velocity intervals are chosen to be the same for all haloes in a catalog. We repeat that procedure for all CDM haloes in a given catalog and finally, for each velocity interval, we compute the mean and the dispersion for all the obtained values. The results are shown in Fig. 13 for four different masses of the host CDM halo: $1 \times 10^{11}$, $1 \times 10^{12}, 1 \times 10^{13}$ and $1 \times 10^{14} h^{-1} \mathrm{M}_{\odot}$, and for two neutrino masses, $\Sigma_{i} m_{\nu_{i}}=0.30$ and $0.60 \mathrm{eV}$. We also compute the velocity distribution for the CDM particles within the CDM halo virial radius and show the results as solid red curves. The error bars we show in that figure represent the $1 \sigma$ error in the estimation of the average distribution while with dashed lines we plot the unperturbed neutrino momentum distribution as given by equation 3.1. In particular, the results have been extracted from the simulations L60, L30, S60 and S30, for neutrinos with masses $\Sigma_{i} m_{\nu_{i}}=0.60$ and $0.30 \mathrm{eV}$, respectively, and from L0 and S30 for the CDM particles distribution.

We find that the neutrino velocity distribution within CDM haloes is, for all CDM halo masses, closer to its unperturbed distribution for lower neutrino masses. This is because the neutrino clustering becomes smaller for lower neutrino masses. On the other hand, since the neutrino clustering increases with the mass of its host CDM halo, the deviation of the neutrino velocity distribution to its unperturbed distribution increases for more massive CDM haloes. Furthermore, since the clustering of neutrinos is larger for more massive neutrinos, the velocity distribution deviates more from its unperturbed distribution in the case of neutrinos with $\Sigma_{i} m_{\nu_{i}}=0.60 \mathrm{eV}$ (in comparison with the $0.30 \mathrm{eV}$ case). In all cases, we find that neutrino velocities are, on average, larger than those from the CDM. The high velocity tail is very well reproduced by the unperturbed velocity distribution. This is not surprising since the dynamics of neutrinos with large velocities is not strongly affected by gravity as can be also seen from Fig. 5. These trends were also obtained by [19] when computing the neutrino momentum distribution at the Earth neighborhood.

\section{Conclusions}

In this paper we have investigated the non-linear evolution of the cosmic neutrino background. We have used a modified version of GADGET-3 that incorporates neutrinos as an independent particle species. Since neutrino 

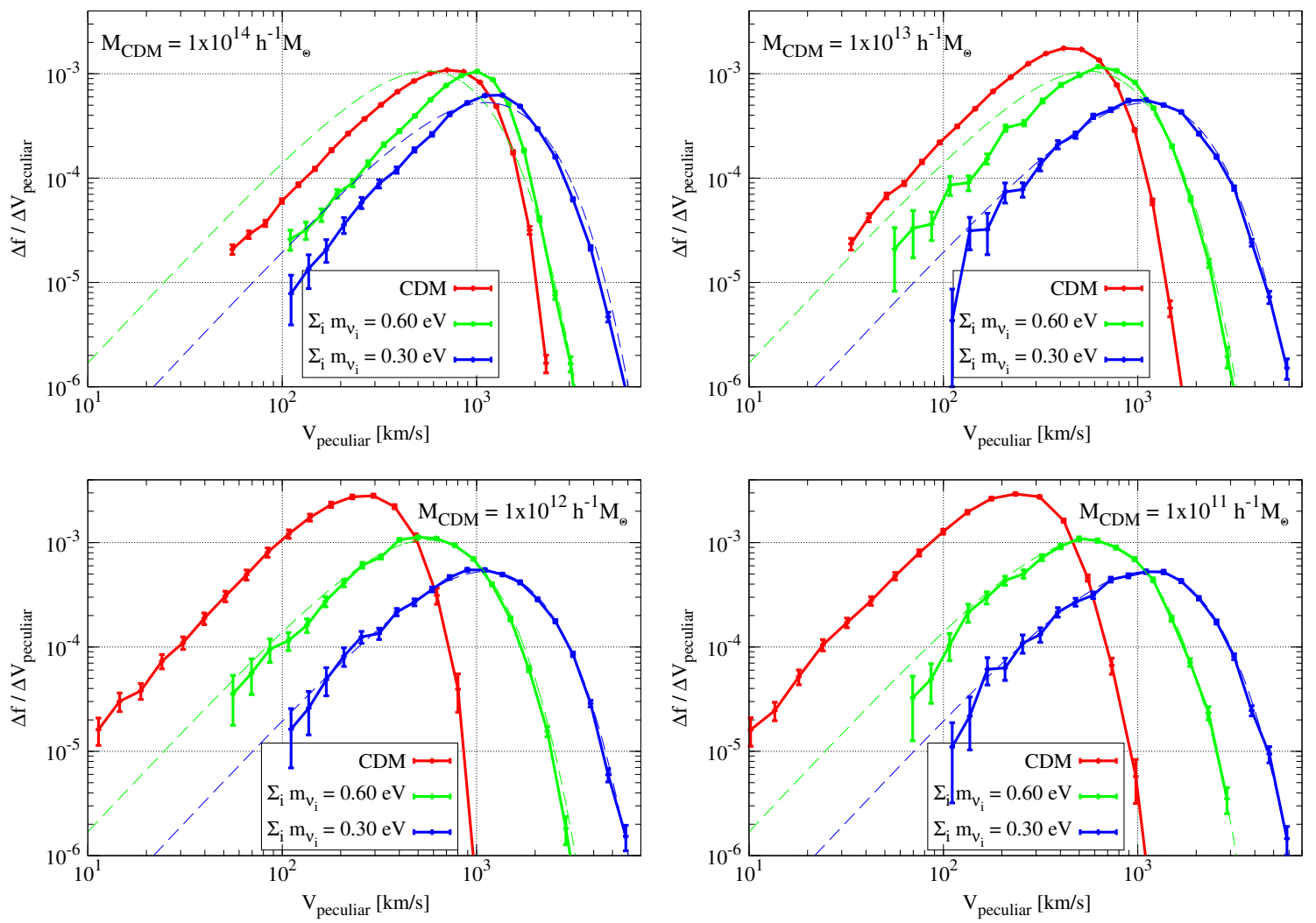

Figure 13. Average CDM and neutrino velocity distribution within the virial radius of CDM haloes. We plot the proportion of particles with peculiar velocities between $V$ and $V+\Delta V$, per $\Delta V$, as a function of the modulus of the peculiar velocity. With solid lines we show the average velocity distribution within CDM haloes with masses equal to $1 \times 10^{14}$ (upper left), $1 \times 10^{13}$ (upper right), $1 \times 10^{12}$ (bottom left) and $1 \times 10^{11}$ (bottom right) $h^{-1} \mathrm{M}_{\odot}$ at $z=0$, for the CDM particles (solid red line), neutrinos with $\Sigma_{i} m_{\nu_{i}}=0.60 \mathrm{eV}$ (green line) and neutrinos with $\Sigma_{i} m_{\nu_{i}}=0.30 \mathrm{eV}$ (blue line). The error bars represent the $1 \sigma$ uncertainty in the average velocity distribution. The dashed lines represent the unperturbed neutrino velocity distribution 3.1.

clustering is expected to be very small and neutrino peculiar velocities are large, it is crucial to investigate resolution, box-size effects and the initial redshift of the simulations (as done by e.g. [11, 13, 41]). We present quantitative results for the following quantities: redshift evolution of the neutrino and CDM density fields over the cosmological volume; redshift evolution of the neutrino and CDM peculiar velocity fields over the cosmological volume; halo mass function in neutrino cosmologies for a large range of halo masses; neutrino properties inside virialized haloes (peculiar velocity and density profiles); neutrino properties outside the virial radius for isolated and not isolated haloes.

Our results can be summarized as follows.

- In the cosmological volume the non-linear CDM and neutrino density fields evolve differently with cosmic time. Whereas the CDM density field evolve quickly, the neutrino density field evolves slowly and is mainly driven by the clustering of neutrinos within CDM halo potential wells.

- The neutrino momentum distribution within the cosmological volume deviates with respect to the unperturbed momentum distribution. These deviations increase with both $\Sigma_{i} m_{\nu_{i}}$ and $a=1 /(1+z)$. At $z=0$, the fraction of neutrinos $\left(\Sigma_{i} m_{\nu_{i}}=0.60 \mathrm{eV}\right)$ with peculiar velocities smaller than $100 \mathrm{~km} / \mathrm{s}$ is a factor two smaller than the one predicted by the unperturbed momentum distribution.

- If we follow the neutrinos in momentum bins, neutrinos with $\Sigma_{i} m_{\nu_{i}}=0.60 \mathrm{eV}$ that have peculiar 
velocities of $\sim 1 / 20$ and $\sim 1 / 10$ of the mean cosmic peculiar velocity in the initial conditions start to behave like CDM at $z=3$ and $z=2$, respectively.

- We computed the halo mass function over four decades in mass and found a reasonably good agreement with the ST mass function by using $\Omega_{\mathrm{M}}=\Omega_{\mathrm{cdm}}+\Omega_{\mathrm{b}}$, without the $\Omega_{\nu}$ contribution.

- We analysed the neutrino density profiles around CDM haloes. As expected, we found the presence of a large core for the most massive haloes above $10^{13.5} h^{-1} \mathrm{M}_{\odot}$ : we provided a simple fitting formula, whose parameter values depend on neutrino mass, cold dark matter mass and redshift.

- For less massive haloes, the resolution of our N-body simulations do not allow us to probe the regime at which the core develops and thereby we present a simpler power-law fitting function to the density profile. For Milky Way size haloes, at $z=0$, the relic neutrino density at the solar radius would be enhanced, with respect to the neutrino background density, by more than a $\sim 40 \%$ for neutrinos with $\Sigma_{i} m_{\nu_{i}}=0.60$ and by more than $\sim 10 \%$ for neutrinos with $\Sigma_{i} m_{\nu_{i}}=0.30$.

- We also considered the peculiar velocity distribution of neutrino particles inside virialized haloes and compared this with the unperturbed Fermi-Dirac distribution. Important deviations take place in the low velocity tail: for neutrinos with $\Sigma_{i} m_{\nu_{i}}=0.60 \mathrm{eV}$ within CDM halos of masses $10^{14} h^{-1} \mathrm{M}_{\odot}$, the fraction of those with velocities lower than $\sim 200 \mathrm{~km} / \mathrm{s}$, is more than a factor six smaller in the real distribution in comparison with the unperturbed distribution.

An accurate modelling and analysis of the impact of relic neutrinos on cosmic structure in the non-linear regime is thus very important. In fact, strong and weak lensing observations of galaxy clusters are already able to place constraints on the density profiles, concentration and shape of these objects (see for example [60, 61]). In this work we have shown that a cored neutrino halo should be present around massive clusters and must impact at some level on the overall cluster properties in a mass and redshift dependent way. Moreover, both spectroscopic and photometric surveys of galaxies can probe the clustering of matter in a region that is affected by the non-linearities of the neutrino component (e.g. [62]). Future large scale structure surveys like Euclid $[35,36,63]$ are thus expected to place tight constraints on neutrino properties by using clustering and weak lensing observations of galaxies and galaxy clusters.

\section{Acknowledgements}

Calculations for this paper were performed on SOM1 and SOM2 at IFIC and on the COSMOS Consortium supercomputer within the DiRAC Facility jointly funded by STFC, the Large Facilities Capital Fund of BIS and the University of Cambridge, as well as the Darwin Supercomputer of the University of Cambridge High Performance Computing Service (http://www.hpc.cam.ac.uk/), provided by Dell Inc. using Strategic Research Infrastructure Funding from the Higher Education Funding Council for England. We thank Volker Springel for giving us permission to use GADGET-3. FVN is supported by the ERC Starting Grant "cosmoIGM". SB is supported by NSF grant AST-0907969 and the IAS. MV acknowledges support from grants: INFN/PD51, ASI/AAE, PRIN MIUR, PRIN INAF 2009 "Towards an Italian Network for Computational Cosmology" and from the ERC Starting Grant "cosmoIGM". The authors wish to thank the referee for the report.

\section{A Impact of the CDM halo environment}

In this paper we have studied the non-linear properties of relic neutrinos within isolated CDM haloes. Here, we investigate how those properties change when considering non-isolated CDM halos. In Sec. 3.4 we defined a CDM halo as isolated if there were no more massive CDM halos located within a distance equal to 10 times the virial radius. If the former condition is not satisfied, then the CDM halo is non-isolated.

In Fig. 14 we show the average neutrino overdensity profiles, at $z=0$, computed within isolated CDM haloes (red), non-isolated CDM haloes (green) and within all CDM haloes (blue). The masses of their host CDM haloes are equal to $10^{14} h^{-1} \mathbf{M}_{\odot}$ (upper left panel), $10^{13} h^{-1} \mathrm{M}_{\odot}$ (upper right panel), $10^{12} h^{-1} \mathrm{M}_{\odot}$ (bottom left panel) and $10^{11} h^{-1} \mathrm{M}_{\odot}$ (bottom right panel) at $z=0$. Each panel shows the overdensity profiles 

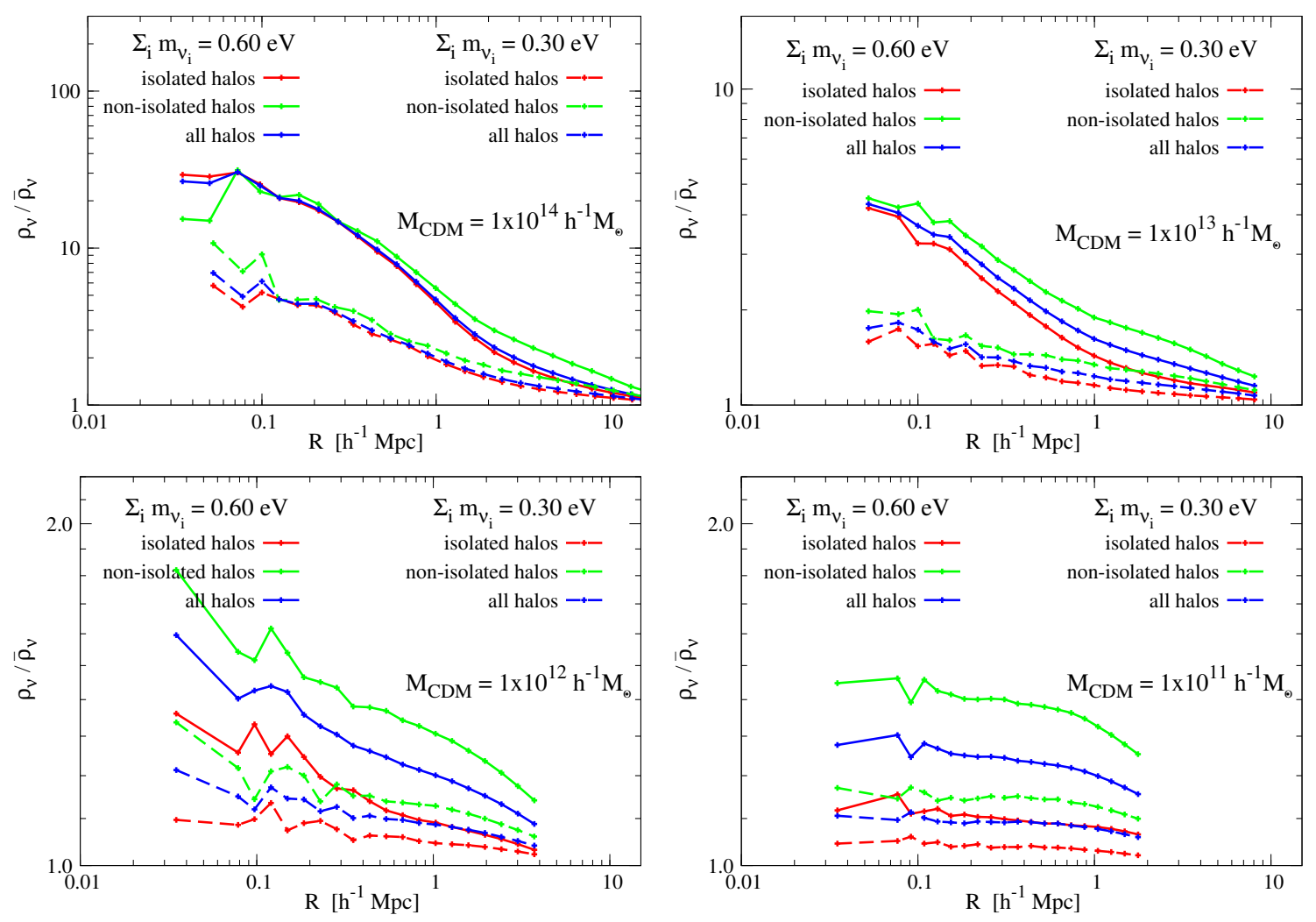

Figure 14. Dependence of the average neutrino overdensity profiles with the environment of their host CDM haloes. The upper left panel shows the neutrino overdensity profiles within CDM haloes of masses equal to $10^{14} h^{-1} \mathbf{M}_{\odot}$, for neutrinos with $\Sigma_{i} m_{\nu_{i}}=0.60 \mathrm{eV}$ (solid lines) and $\Sigma_{i} m_{\nu_{i}}=0.30 \mathrm{eV}$ (dashed lines). The profiles are computed within isolated CDM haloes (red lines), non-isolated CDM haloes (green lines) and all haloes (blue lines). The other panels show the same for $\mathrm{CDM}$ halo masses equal to $10^{13} h^{-1} \mathrm{M}_{\odot}$ (upper right), $10^{12} h^{-1} \mathrm{M}_{\odot}$ (bottom left) and $10^{11} h^{-1} \mathrm{M}_{\odot}$ (bottom right).

\begin{tabular}{|l||c|c|c|}
\hline & Isolated haloes & Non-Isolated haloes & All haloes \\
\hline \hline$\Sigma_{i} m_{\nu_{i}}=0.60 \mathrm{eV}: \mathrm{M}_{\mathrm{CDM}}=10^{14} \mathrm{M}_{\odot}$ & 205 & 49 & 254 \\
\hline$\Sigma_{i} m_{\nu_{i}}=0.30 \mathrm{eV}: \mathrm{M}_{\mathrm{CDM}}=10^{14} \mathrm{M}_{\odot}$ & 275 & 86 & 361 \\
\hline$\Sigma_{i} m_{\nu_{i}}=0.60 \mathrm{eV}: \mathrm{M}_{\mathrm{CDM}}=10^{13} \mathrm{M}_{\odot}$ & 2389 & 1637 & 4026 \\
\hline$\Sigma_{i} m_{\nu_{i}}=0.30 \mathrm{eV}: \mathrm{M}_{\mathrm{CDM}}=10^{13} \mathrm{M}_{\odot}$ & 2495 & 1798 & 4293 \\
\hline$\Sigma_{i} m_{\nu_{i}}=0.60 \mathrm{eV}: \mathrm{M}_{\mathrm{CDM}}=10^{12} \mathrm{M}_{\odot}$ & 171 & 178 & 349 \\
\hline$\Sigma_{i} m_{\nu_{i}}=0.30 \mathrm{eV}: \mathrm{M}_{\mathrm{CDM}}=10^{12} \mathrm{M}_{\odot}$ & 169 & 160 & 329 \\
\hline$\Sigma_{i} m_{\nu_{i}}=0.60 \mathrm{eV}: \mathrm{M}_{\mathrm{CDM}}=10^{11} \mathrm{M}_{\odot}$ & 1362 & 1266 & 2628 \\
\hline$\Sigma_{i} m_{\nu_{i}}=0.30 \mathrm{eV}: \mathrm{M}_{\mathrm{CDM}}=10^{11} \mathrm{M}_{\odot}$ & 1434 & 1354 & 2788 \\
\hline
\end{tabular}

Table 2. Number of CDM haloes found in the simulations depending on their masses, on their environment and on the masses of the neutrinos. CDM haloes with masses equal or larger than $10^{13} h^{-1} \mathrm{M}_{\odot}$ are extracted from the simulations L60 and L30, whereas the rest are extracted from the simulations S60 and S30.

for two neutrino masses: $\Sigma_{i} m_{\nu_{i}}=0.60 \mathrm{eV}$ (solid lines) and $\Sigma_{i} m_{\nu_{i}}=0.30 \mathrm{eV}$ (dashed lines). In particular, the results shown have been extracted from the simulations L60 and L30, for CDM halo masses equal or larger than $1 \times 10^{13} h^{-1} \mathrm{M}_{\odot}$, and from the simulations S60 and S30 for CDM halo masses smaller than $1 \times 10^{13}$ $h^{-1} \mathbf{M}_{\odot}$. Table 2 shows the number of CDM haloes for each neutrino mass, each CDM halo mass and for each halo environment. 

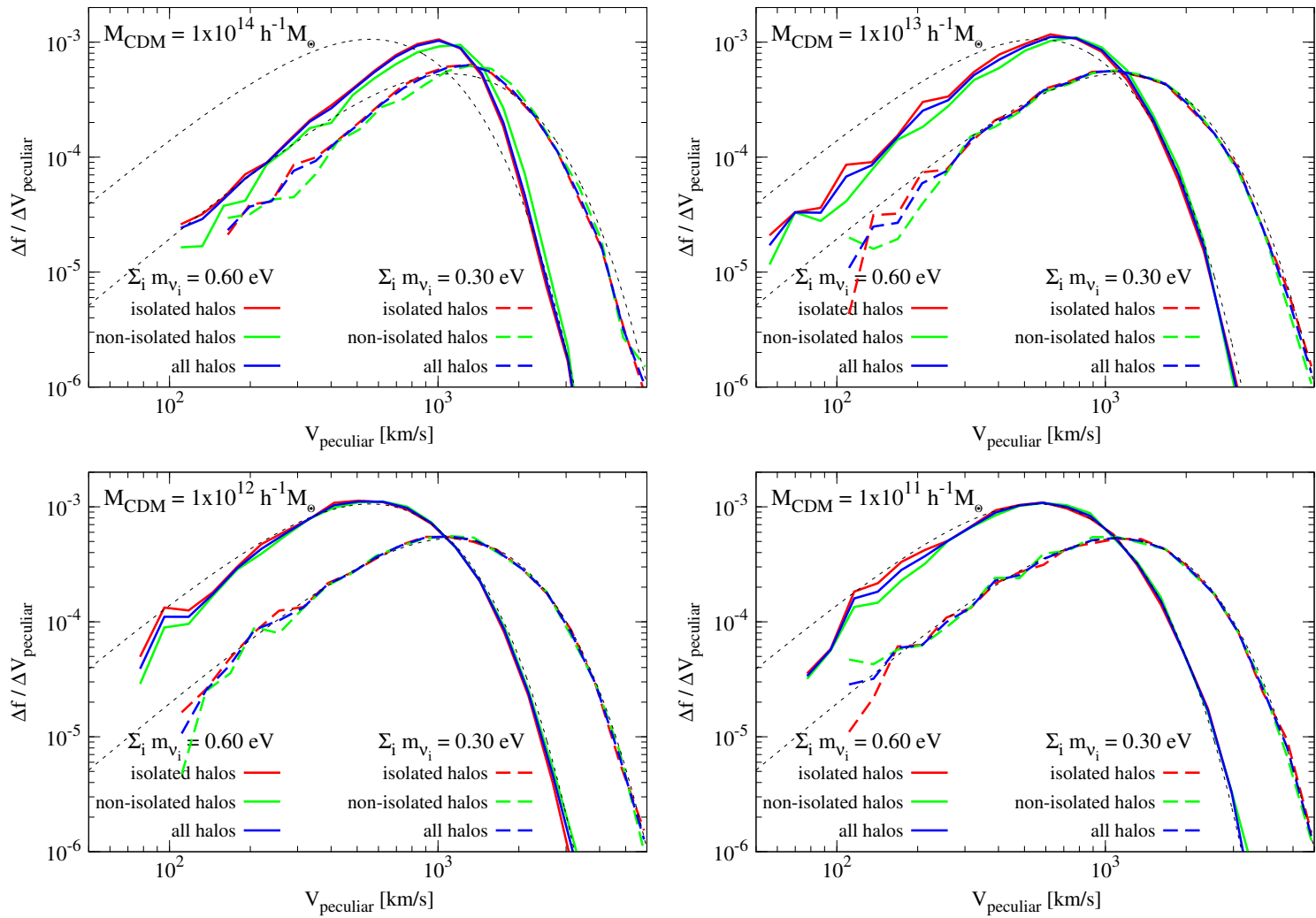

Figure 15. Dependence of the distribution of the neutrino peculiar velocities with the environment of their host CDM halo. We show the proportion of neutrinos, within the virial radius of their host CDM haloes, with peculiar velocities between $V$ and $V+\triangle V$, per $\triangle V$, as a function of the neutrino peculiar velocity modulus for different CDM halo environments: isolated CDM haloes (red), non-isolated CDM haloes (green) and all CDM haloes (blue). Two neutrino masses, $\Sigma_{i} m_{\nu_{i}}=0.60 \mathrm{eV}$ (solid lines) and $\Sigma_{i} m_{\nu_{i}}=0.30 \mathrm{eV}$ (thick long-dashed lines), are studied within CDM haloes of masses equal to $10^{14} h^{-1} \mathrm{M}_{\odot}$ (upper left panel), $10^{13} h^{-1} \mathrm{M}_{\odot}$ (upper right panel), $10^{12} h^{-1} \mathrm{M}_{\odot}$ (bottom left panel) and $10^{11} h^{-1} \mathrm{M}_{\odot}$ (bottom right panel). The black thin short-dashed lines show the results of the unperturbed neutrino distribution as given by Eq. 3.1.

We find that the clustering of neutrinos is larger within non-isolated CDM halos than within isolated CDM halos. This is not surprising since we expect higher values in the density profile due to the presence of a heavier halo in the vicinity of non-isolated CDM haloes. The presence of the heavier halo may only locally modify the density prole, (e.g. when the halo is very far away), or it could inuence the overall prole. The last situation corresponds to the case in which, for example, the halo is a satellite of a much heavier halo. In that case, the majority of the relic neutrinos will be orbiting around the heavier halo, and therefore, the neutrino density profile around the halo into study will be completely distorted since it will be embedded into a larger and denser neutrino halo. As expected, we find that the dispersion in the neutrino density profile within non-isolated CDM haloes is larger than within isolated CDM haloes.

It turns out that the fitting formulas 3.4 and 3.5 provide an excellent description of the neutrino density profiles, independently of the environment of their host CDM haloes. The environment of the CDM haloes affects, of course, the values of the fitting formula parameters.

Finally, in Fig. 15, we investigate the impact of the CDM halo environment on the distribution of neutrino peculiar velocities within the virial radius of CDM haloes. We examine a range of halo and neutrino masses in three different environments: isolated CDM haloes, non-isolated CDM haloes and all haloes.

Since we are focusing on the neutrino peculiar velocity distribution within the CDM halo virial radius, it is expected that the CDM halo environment does not play a critical role in the results. The presence of a more 


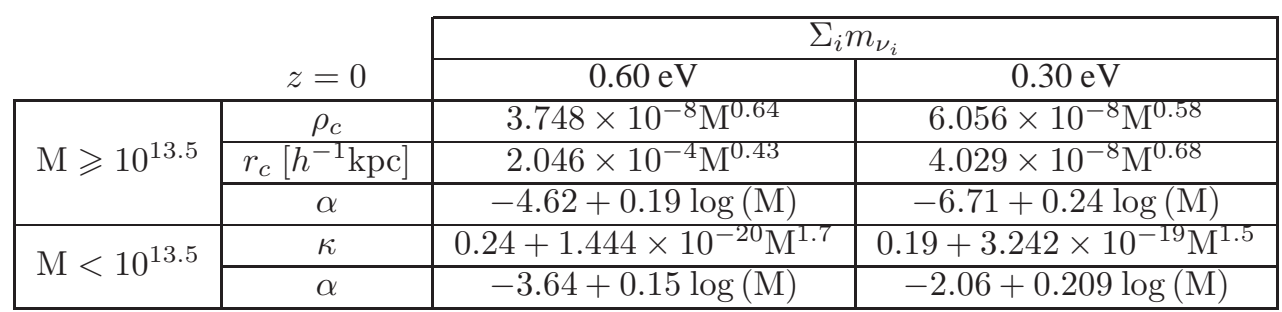

Table 3. Formulae that reproduce the values of the parameters of the fitting formulas 3.4 and 3.5 over a wide range of masses as shown in Fig. 10. We have defined $\mathrm{M}=\mathrm{M}_{\mathrm{CDM}} /\left(h^{-1} \mathrm{M}_{\odot}\right)$.

massive CDM halo can only significantly modify the distribution of neutrino velocities within the CDM halo virial radius if it is close enough. If this is the case, then, as we find, non-isolated CDM haloes will contain a smaller fraction of neutrinos with low velocities. This is because the proportion of neutrinos with large velocities, some of them belonging to a larger and deeper neutrino halo centered in the more massive CDM halo, is enhanced for non-isolated CDM halos due to the presence of a heavier CDM halo in their neighborhood.

\section{B Fitting formula parameters: dependence with $M_{\mathrm{CDM}}$ and $\Sigma_{i} m_{\nu_{i}}$}

In table 3 we show the preferred values for the parameters of equations 3.4 and 3.5 over a wide range of CDM halo masses. They are presented for two neutrino masses: $\Sigma_{i} m_{\nu_{i}}=0.30 \mathrm{eV}$ and $\Sigma_{i} m_{\nu_{i}}=0.60 \mathrm{eV}$. The parameter $\kappa$ in equation 3.5 is dimensionless. Thus for the above formulae the distance $r$ in 3.5 is assumed to be in $h^{-1} \mathrm{kpc}$.

\section{References}

[1] E. W. Kolb and M. S. Turner, Front. Phys. 69, 1 (1990).

[2] S. Dodelson, Amsterdam, Netherlands: Academic Pr. (2003) 440 p

[3] J. Lesgourgues and S. Pastor, Phys. Rept. 429, 307 (2006) [astro-ph/0603494].

[4] S. Weinberg, Oxford, UK: Oxford Univ. Pr. (2008) 593 p

[5] G. Mangano, G. Miele, S. Pastor, T. Pinto, O. Pisanti and P. D. Serpico, Nucl. Phys. B 729, 221 (2005) [hep-ph/0506164].

[6] C. -P. Ma and E. Bertschinger, Astrophys. J. 429, 22 (1994) [astro-ph/9308006].

[7] R. Valdarnini, T. Kahniashvili and B. Novosyadlyj, Astron. Astrophys. 336, 11 (1998) [astro-ph/9804057].

[8] J. Brandbyge, S. Hannestad, T. Haugbolle and B. Thomsen, JCAP 0808, 020 (2008) [arXiv:0802.3700 [astro-ph]].

[9] J. Brandbyge and S. Hannestad, JCAP 0905, 002 (2009) [arXiv:0812.3149 [astro-ph]].

[10] J. Brandbyge and S. Hannestad, JCAP 1001, 021 (2010) [arXiv:0908.1969 [astro-ph.CO]].

[11] M. Viel, M. G. Haehnelt and V. Springel, JCAP 1006, 015 (2010) [arXiv:1003.2422 [astro-ph.CO]].

[12] S. Agarwal and H. A. Feldman, Mon. Not. Roy. Astron. Soc. 410, 1647 (2011) [arXiv:1006.0689 [astro-ph.CO]].

[13] S. Bird, M. Viel and M. G. Haehnelt, Mon. Not. Roy. Astron. Soc. 420, 2551 (2012) [arXiv:1109.4416 [astro-ph.CO]].

[14] C. Wagner, L. Verde and R. Jimenez, arXiv:1203.5342 [astro-ph.CO].

[15] Y. Ali-Haimoud and S. Bird, arXiv:1209.0461 [astro-ph.CO].

[16] S. Saito, M. Takada and A. Taruya, Phys. Rev. D 83, 043529 (2011) [arXiv:1006.4845 [astro-ph.CO]].

[17] J. Lesgourgues, S. Matarrese, M. Pietroni and A. Riotto, JCAP 0906, 017 (2009) [arXiv:0901.4550 [astro-ph.CO]].

[18] S. Singh and C. -P. Ma, Phys. Rev. D 67, 023506 (2003) [astro-ph/0208419].

[19] A. Ringwald and Y. Y. Y. Wong, JCAP 0412, 005 (2004) [hep-ph/0408241]. 
[20] F. Villaescusa-Navarro, J. Miralda-Escude, C. Pena-Garay and V. Quilis, JCAP 1106, 027 (2011) [arXiv: 1104.4770 [astro-ph.CO]].

[21] K. Abazajian, E. R. Switzer, S. Dodelson, K. Heitmann and S. Habib, Phys. Rev. D 71, 043507 (2005) [astro-ph/0411552].

[22] K. Ichiki, M. Takada Phys. Rev. D 85, 063521 (2012) [arXiv:1108.4688 [astro-ph.CO]].

[23] M. Shoji, E. Komatsu Phys. Rev. D 81, 123516 (2010).

[24] M. C. Gonzalez-Garcia, M. Maltoni, J. Salvado and T. Schwetz, arXiv:1209.3023 [hep-ph].

[25] B.A. Reid, L. Verde, R. Jimenez, O. Mena, JCAP 01, 003 (2010) [arXiv:0910.0008 [astro-ph.CO]].

[26] S.A. Thomas; F.B. Abdalla; O. Lahav Phys. Rev. Lett. 105, 031301 (2010) [arXiv:0911.5291 [astro-ph.CO]].

[27] M. E. C. Swanson, W. J. Percival and O. Lahav, Mon. Not. Roy. Astron. Soc. 409, 1100 (2010) [arXiv:1006.2825 [astro-ph.CO]].

[28] R. de Putter, O. Mena, E. Giusarma, S. Ho, A. Cuesta, H. -J. Seo, A. Ross and M. White et al., Astrophys. J. 761, 12 (2012) [arXiv:1201.1909 [astro-ph.CO]].

[29] J. -Q. Xia, B. R. Granett, M. Viel, S. Bird, L. Guzzo, M. G. Haehnelt, J. Coupon and H. J. McCracken et al., JCAP 1206, 010 (2012) [arXiv:1203.5105 [astro-ph.CO]].

[30] S. Riemer-Sorensen, C. Blake, D. Parkinson, T. M. Davis, S. Brough, M. Colless, C. Contreras and W. Couch et al., Phys. Rev. D 85, 081101 (2012) [arXiv:1112.4940 [astro-ph.CO]].

[31] G. -B. Zhao, S. Saito, W. J. Percival, A. J. Ross, F. Montesano, M. Viel, D. P. Schneider and D. J. Ernst et al., arXiv:1211.3741 [astro-ph.CO].

[32] U. Seljak, A. Slosar and P. McDonald, JCAP 0610, 014 (2006) [astro-ph/0604335].

[33] K. T. Story, C. L. Reichardt, Z. Hou, R. Keisler, K. A. Aird, B. A. Benson, L. E. Bleem and J. E. Carlstrom et al., arXiv:1210.7231 [astro-ph.CO].

[34] P. A. R. Ade et al. [Planck Collaboration], Astron. Astrophys. 536, 16464 (2011) [arXiv:1101.2022 [astro-ph.IM]].

[35] J. Hamann, S. Hannestad and Y. Y. Y. Wong, JCAP 1211, 052 (2012) [arXiv:1209.1043 [astro-ph.CO]].

[36] B. Audren, J. Lesgourgues, S. Bird, M. G. Haehnelt and M. Viel, arXiv:1210.2194 [astro-ph.CO].

[37] R. Jimenez, T. Kitching, C. Pena-Garay, L. Verde, JCAP 05, 035 (2010) [arXiv:1003.5918 [astro-ph.CO]]

[38] M. Takada, E. Komatsu, T. Futamase, Phys. Rev. D 73, 083520 (2006) [arXiv:astro-ph/0512374].

[39] J. J. Gomez-Cadenas, J. Martin-Albo, M. Mezzetto, F. Monrabal and M. Sorel, Riv. Nuovo Cim. 35, 29 (2012) [arXiv:1109.5515 [hep-ex]].

[40] B. Kayser, J. Phys. Conf. Ser. 173, 012013 (2009) [arXiv:0903.0899 [hep-ph]].

[41] J. Brandbyge, S. Hannestad, T. Haugboelle and Y. Y. Y. Wong, JCAP 1009, 014 (2010) [arXiv:1004.4105 [astro-ph.CO]].

[42] V. Springel and L. Hernquist, Mon. Not. Roy. Astron. Soc. 339, 289 (2003) [astro-ph/0206393].

[43] R. Scoccimarro, Mon. Not. Roy. Astron. Soc. 299, 1097 (1998) [astro-ph/9711187].

[44] G. Somogyi and R. E. Smith, Phys. Rev. D 81, 023524 (2010) [arXiv:0910.5220 [astro-ph.CO]].

[45] Y. .B. Zeldovich, Astron. Astrophys. 5, 84 (1970).

[46] E. Komatsu et al. [WMAP Collaboration], Astrophys. J. Suppl. 192, 18 (2011) [arXiv:1001.4538 [astro-ph.CO]].

[47] V. Springel, S. D. M. White, G. Tormen and G. Kauffmann, Mon. Not. Roy. Astron. Soc. 328, 726 (2001) [astro-ph/0012055].

[48] W. H. Press and P. Schechter, Astrophys. J. 187, 425 (1974).

[49] R. K. Sheth and G. Tormen, Mon. Not. Roy. Astron. Soc. 308, 119 (1999) [astro-ph/9901122].

[50] G. Efstathiou, C. S. Frenk, S. D. M. White and M. Davis, Mon. Not. Roy. Astron. Soc. 235, 715 (1988).

[51] A. Jenkins, C. S. Frenk, S. D. M. White, J. M. Colberg, S. Cole, A. E. Evrard, H. M. P. Couchman and N. Yoshida, Mon. Not. Roy. Astron. Soc. 321, 372 (2001) [astro-ph/0005260]. 
[52] J. E. Gunn and J. R. Gott, III, Astrophys. J. 176, 1 (1972).

[53] A. Lewis, A. Challinor and A. Lasenby, Astrophys. J. 538, 473 (2000) [astro-ph/9911177].

[54] G. L. Bryan and M. L. Norman, Astrophys. J. 495, 80 (1998) [astro-ph/9710107].

[55] F. Marulli, C. Carbone, M. Viel, L. Moscardini and A. Cimatti, Mon. Not. Roy. Astron. Soc. 418, 346 (2011) [arXiv:1103.0278 [astro-ph.CO]].

[56] A. E. Evrard, J. Bialek, M. Busha, M. White, S. Habib, K. Heitmann, M. Warren and E. Rasia et al., Astrophys. J. 672, 122 (2008) [astro-ph/0702241 [ASTRO-PH]].

[57] J. F. Navarro, C. S. Frenk and S. D. M. White, Astrophys. J. 490, 493 (1997) [astro-ph/9611107].

[58] S. Tremaine and J. E. Gunn, Phys. Rev. Lett. 42, 407 (1979).

[59] F. Villaescusa-Navarro, M. Vogelsberger, M. Viel and A. Loeb, arXiv:1106.2543 [astro-ph.CO].

[60] K. Umetsu, E. Medezinski, M. Nonino, J. Merten, A. Zitrin, A. Molino, C. Grillo and M. Carrasco et al., Astrophys. J. 755, 56 (2012) [arXiv:1204.3630 [astro-ph.CO]].

[61] A. B. Newman, T. Treu, R. S. Ellis and D. J. Sand, arXiv:1209.1392 [astro-ph.CO].

[62] J. -Q. Xia, B. R. Granett, M. Viel, S. Bird, L. Guzzo, M. G. Haehnelt, J. Coupon and H. J. McCracken et al., JCAP 1206, 010 (2012) [arXiv:1203.5105 [astro-ph.CO]].

[63] R. Laureijs, J. Amiaux, S. Arduini, J. -L. Augueres, J. Brinchmann, R. Cole, M. Cropper and C. Dabin et al., arXiv:1110.3193 [astro-ph.CO]. 